\title{
Robust and Computationally Efficient Signal-Dependent Method for Joint DOA and Frequency Estimation
}

\author{
Ting Shu and Xingzhao Liu \\ Department of Electronic Engineering, Shanghai Jiao Tong University, Shanghai, 200240, China \\ Correspondence should be addressed to Ting Shu, tingshu@sjtu.edu.cn
}

Received 17 September 2007; Revised 29 January 2008; Accepted 12 April 2008

Recommended by Fulvio Gini

The problem of joint direction of arrival (DOA) and frequency estimation is considered in this paper. A new method is proposed based on the signal-dependent multistage wiener filter (MWF). Compared with the classical subspace-based joint DOA and frequency estimators, the proposed method has two major advantages: (1) it provides a robust performance in the presence of colored noise; (2) it does not involve the estimation of covariance matrix and its eigendecomposition, and thus, yields much lower computational complexity. These advantages can potentially make the proposed method more feasible in practical applications. The conditional Cramér-Rao lower bound (CRB) on the error variance for joint DOA and frequency estimation is also derived. Both numerical and experimental results are used to demonstrate the performance of the proposed method.

Copyright (c) 2008 T. Shu and X. Liu. This is an open access article distributed under the Creative Commons Attribution License, which permits unrestricted use, distribution, and reproduction in any medium, provided the original work is properly cited.

\section{INTRODUCTION}

The problem of simultaneously estimating the spatial and temporal frequencies of multiple narrowband plane waves has received considerable attention in the past few decades [1-10]. This problem is crucial in many practical applications, such as array processing, joint angle and Doppler estimation for space-time adaptive processing (STAP) airborne radar, synthetic aperture radar (SAR) imaging, and some electronic warfare and sonar systems. See $[2,5,6,9]$ for a detailed description, $[1,3,7,8]$ for some of the earlier work, and $[9,10]$ for some of more recent work. The joint estimation has a number of advantages. First, as shown in Section 2.1, the number of sources can be significantly larger than the number of antennas by using the spatiotemporal data model. Second, in the spatiotemporal data model, multiple sources with the same DOA can be resolved (see Property 2 in Section 2.1, Figure 5 in Example 1 and Figure 6 in Example 2). Finally, the estimation accuracy can be improved (see Figures 6 and 7 in Example 2, Figure 14 in Section 3.2).

Although the well-known maximum likelihood approaches (see, e.g., $[1,2]$ and the references therein) can provide optimum parameter estimation in the presence of white Gaussian noise, they are perceived to be too computationally complex. Based on the subspace techniques, a number of suboptimal algorithms have been developed, such as multiple signal classification (MUSIC) [11] and estimation of signal parameters via rotational invariance technique (ESPRIT) [12]. Some of these suboptimal algorithms have been used to solve the problem of joint direction of arrival (DOA) and frequency estimation [3-10]. For example, Zoltowski and Mathews [7] have discussed this problem in the electronic warfare applications. To cover a very wide frequency band $(2-18 \mathrm{GHz})$, a nonuniform linear array is used to resolve the angular ambiguity. Their methods are mainly motivated by engineering considerations. Haardt and Nossek [8] have proposed a method for joint 2D angle and frequency estimation based on the Unitary-ESPRIT in the space-division multiple access (SDMA) applications. Viberg and Stocia [6] have presented a prewhitened subspacebased method for joint DOA and frequency estimation in the colored noise. Another ESPRIT-based method called joint angle-frequency estimation (JAFE) has been proposed by Lemma et al. in [4], and it has been considered as the state-of-the-art among suboptimal joint DOA-frequency estimators. The recent work of Lin et al. [9] has proposed a frequency-space-frequency (FSF) MUSIC-based algorithm in wireless communication applications. It is a tree-structure method which can provide a comparable performance to 
the JAFE. Another recent work of Belkacemi and Marcos [10] has discussed the problem of joint angle-Doppler estimation in the presence of impulsive noise and clutter in the airborne radar applications. This method models the impulsive noise and clutter as the so-called symmetric $\alpha$-stable $(\mathrm{S} \alpha \mathrm{S})$ process, and a preprocessing technique called phased fractional lower-order moment (PFLOM) is used before applying the 2-D MUSIC [3] to estimate the angle and Doppler. Generally speaking, these algorithms are known to have high-resolution capabilities and yield accurate estimates. However, there are two major drawbacks in practical applications. First, most existing techniques are under the additive white Gaussian noise assumption [3-5, 79]. Unfortunately, in practice, the noise is often spatially correlated. As a consequence, the colored noise may degrade the performance of these algorithms significantly. In addition, if the noise covariance matrix is known, the spatially colored noise can be prewhitened $[6,11]$. In practice, the noise covariance is often measured experimentally from the signalfree data. However, such signal-free data is often unavailable. Thus, accurate parameter estimation is impossible without good priori knowledge of the colored noise. Secondly, due to the eigendecomposition of the sample covariance matrix or the singular value decomposition (SVD) of the data matrix, the computational burden is often prohibitively extensive in the case of large antenna array systems and multidimensional applications (e.g., array radar systems) where the model order is large. Therefore, for practical considerations, robustness and computational efficiency are always of great importance.

On the other hand, the problem of parameter estimation with a priori knowledge, such as the waveform of the desired signal and the steering vector of the array (or the main beam pattern of the antenna), has been well studied. Li and Compton Jr. [13] and Li et al. [14] have proposed algorithms for DOA estimation with known waveforms. Later, Wax and Leshem [15] and Swindlehurst et al. [16, 17] have discussed the problem of joint parameters estimation with known waveforms, respectively. Recently, Gini et al. [18] have proposed a method of multiple radar targets estimation by exploiting the knowledge of the antenna main beam pattern and induced amplitude modulation. A discussion of their applications to active radar systems, mobile communication systems, ALOHA packet radio systems, and explosive detection can be found in [13-20]. It is demonstrated that exploiting temporal information about the signal can improve the performance of DOA estimation $[13,14,20]$. In this paper, we will show that one cannot only improve the robustness of algorithm but also reduce the computational complexity by using a priori knowledge of one desired signal.

The main contribution of this paper can be briefly stated as follows: applying the signal-dependent multistage Wiener filter (MWF) technique [21] so as to accurately determine the signal subspace even when the noise background is both spatially and temporally colored. The MWF is a reducedrank adaptive filtering technique that has been used in the application of reduced-rank STAP for airborne radar [22] and the suppression of multiple-access interference for mobile communication [23]. In this paper, we introduce it to joint DOA and frequency estimation. The motivation of applying the MWF lies in its inherent robustness to eigenspectrum spreading (referred to as the subspace leakage problem [24]). (Eigenspectrum spreading refers to an increase in the number of interference eigenvalues of the covariance matrix due to a multitude of real-world effects. In practice, eigenspectrum spreading is always present particularly in the colored noise environment.) Moreover, by using the MWF, the proposed method does not need the estimation of the covariance matrix and its eigendecomposition, and hence, it is more computationally efficient than the classical subspace-based methods. Before presenting the numerical results, the conditional Cramér-Rao lower bound (CRB) on the parameter estimation is derived. Our new expressions of CRB can be viewed as an extension of the well-known results of Stoica et al. Then, the performance of the proposed method is demonstrated by using both numerical and experimental examples.

The remainder of this paper is organized as follows. In Section 2, first we describe the data model and some necessary preliminaries. Then, the proposed method and the conditional CRB on the parameter estimation are presented. Section 3 shows numerical and experimental results, and Section 4 concludes the paper.

The following notations are used throughout this paper. Superscripts $(\cdot)^{T},(\cdot)^{*},(\cdot)^{H},(\cdot)^{\#}, \otimes$, and $\odot$ denote the operation of transpose, complex conjugate, complex conjugate transpose, pseudoinverse, the Kronecker product and the Hardamard product, respectively. The notation diag[a] denotes a diagonal matrix with its diagonal elements formed by vector a. The notation $\|\mathbf{a}\|$ denotes the Euclidian norm of vector $\mathbf{a}$. The notation $\|\mathbf{A}\|_{F}$ denotes the Frobenius norm of matrix A. The notation $\angle(\cdot)$ denotes the phase angle. The notation $E[\cdot]$ denotes the expectation of a random variable. $\mathbf{P}_{\boldsymbol{\Delta}}=\boldsymbol{\Delta}\left(\boldsymbol{\Delta}^{H} \boldsymbol{\Delta}\right)^{-1} \boldsymbol{\Delta}^{H}$ and $\mathbf{P}_{\Delta}^{\perp}=\mathbf{I}-\mathbf{P}_{\boldsymbol{\Delta}}$ stand for the orthogonal projection matrices onto the space of $\Delta$ and its orthogonal complement.

\section{PROBLEM FORMULATION}

\subsection{Data model}

Consider a uniform linear array (ULA) with $M$ elements. Impingings on the array are $P$ narrowband plane waves, which indicates that the effect of a time delay on the received waveform is a phase shift. Let $\omega_{c}$ be the center frequency of the band of interest, and suppose that the $i$ th $(i=$ $1,2, \ldots, P)$ source comes from a direction of $\theta_{i}$. Thus, after demodulation to baseband or intermediate frequency (IF), the output of ULA at time $t$ can be written as

$$
\mathbf{x}(t)=\sum_{i=1}^{P} \mathbf{a}\left(\theta_{i}\right) \alpha_{i} p_{i}(t) e^{j \omega_{i} t}+\mathbf{n}(t), \quad t=0,1, \ldots, N-1
$$

where $\omega_{i}, p_{i}(t)$, and $\alpha_{i}$ denote the baseband frequency after sampling, the waveform, and the complex amplitude of the $i$ th source, respectively. $\mathbf{a}\left(\theta_{i}\right)$ is the $M \times 1$ spatial steering vector of the array toward direction $\theta_{i}$ and $\mathbf{n}(t)$ is the $M \times 1$ 


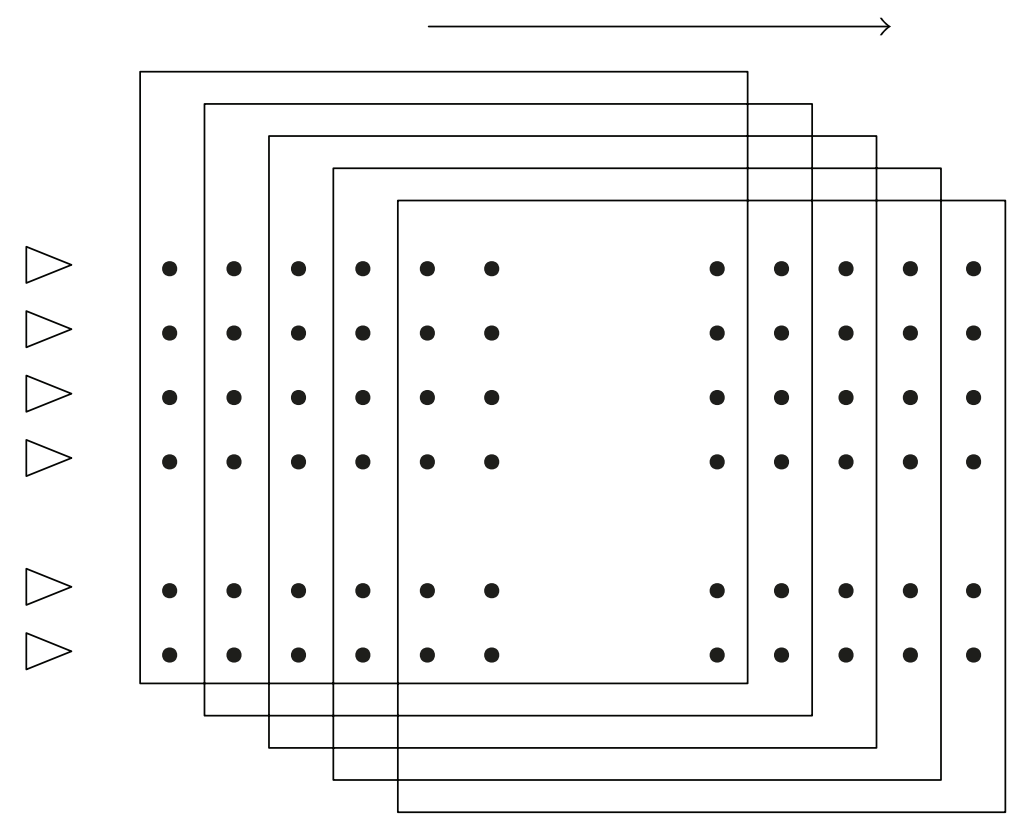

FIgURE 1: Data stacking technique $(K=5)$.

noise vector. For ULA, the spatial steering vector $\mathbf{a}\left(\theta_{i}\right)$ has the form

$$
\mathbf{a}\left(\theta_{i}\right)=\left[1, e^{j 2 \pi d \sin \theta_{i} / \lambda_{i}}, \ldots, e^{j 2 \pi(M-1) d \sin \theta_{i} / \lambda_{i}}\right]^{T},
$$

where $d$ and $\lambda_{i}$ are the interelement spacing and the wavelength of the $i$ th source, respectively.

Next, we define the $M \times P$ steering matrix (referred to as the array manifold) $\mathbf{A}$, the $P \times 1$ signal vector $\mathbf{s}(t)$, and the $P \times P$ diagonal matrix $\Phi$ as

$$
\begin{aligned}
\mathbf{A} & =\left[\mathbf{a}\left(\theta_{1}\right), \ldots, \mathbf{a}\left(\theta_{P}\right)\right], \\
\mathbf{s}(t) & =\left[\alpha_{1} p_{1}(t), \ldots, \alpha_{P} p_{P}(t)\right]^{T}, \\
\boldsymbol{\Phi} & =\operatorname{diag}\left[e^{j \omega_{1}}, \ldots, e^{j \omega_{P}}\right] .
\end{aligned}
$$

Note that $\Phi$ is the diagonal matrix only containing information about the temporal frequency $\omega_{i}$. Then, the array output can be expressed as

$$
\mathbf{x}(t)=\mathbf{A} \Phi^{t} \mathbf{s}(t)+\mathbf{n}(t) .
$$

After that, we use the data stacking technique (referred to as temporal smoothing [5]) to create the spatiotemporal data matrix (see Figure 1). By stacking $K$ (referred to as temporal smoothing factor) temporal shifted versions of the original array output matrix, we have the following $M K \times(N-K+1)$ spatiotemporal data matrix:

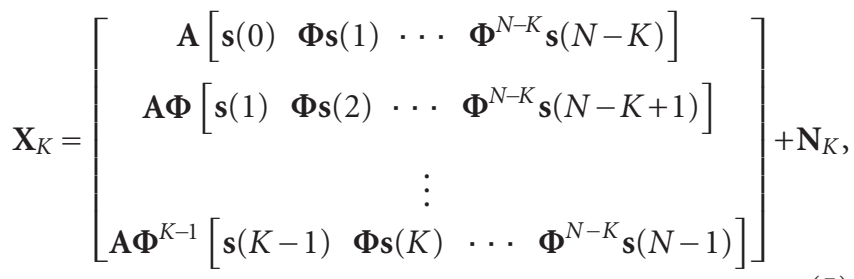

where $\mathbf{N}_{K}$ is the $M K \times(N-K+1)$ temporally smoothed noise matrix which has the same form of $\mathbf{X}_{K}$. With the narrowband assumption, we have $\mathbf{s}(t) \approx \mathbf{s}(t+1) \approx \cdots \approx \mathbf{s}(t+K-1)$. Then, the spatiotemporal data matrix in (5) can be expressed as follows:

$$
\begin{aligned}
\mathbf{X}_{K} & =\left[\begin{array}{c}
\mathbf{A} \\
\mathbf{A} \boldsymbol{\Phi} \\
\vdots \\
\mathbf{A} \boldsymbol{\Phi}^{K-1}
\end{array}\right]\left[\begin{array}{llll}
\mathbf{s}(0) & \boldsymbol{\Phi} \mathbf{s}(1) & \cdots & \boldsymbol{\Phi}^{N-K} \mathbf{s}(N-K)
\end{array}\right]+\mathbf{N}_{K} \\
& =\boldsymbol{\Omega} \mathbf{S}_{K}+\mathbf{N}_{K},
\end{aligned}
$$

where $\mathbf{S}_{K}$ is the $P \times(N-K+1)$ matrix, and $\boldsymbol{\Omega}=$ $\left[\mathbf{A}^{T},(\mathbf{A} \Phi)^{T}, \ldots,\left(\mathbf{A} \Phi^{K-1}\right)^{T}\right]^{T}$ is the $M K \times P$ matrix (referred to as the spatiotemporal manifold) whose range space plays the role of spatiotemporal signal subspace. It fact, the spatiotemporal data can be obtained without performing the data stacking in some applications (see the discussion in Section 2.2), then, (6) can be rewritten in a form of snapshot vector

$$
\mathbf{X}_{K}(t)=\boldsymbol{\Omega} \boldsymbol{\Phi}^{t} \mathbf{s}(t)+\mathbf{N}_{K}(t), \quad t=0,1, \ldots, N-1 .
$$

Property 1. Let $\mathbf{b}\left(\omega_{i}\right)=\left[1, e^{j \omega_{i}}, \ldots, e^{j(K-1) \omega_{i}}\right]^{T}$ denote the $K \times 1$ temporal steering vector. Then, $\boldsymbol{\Omega}$ can be expressed as $\boldsymbol{\Omega}=\left[\boldsymbol{\Xi}\left(\theta_{1}, \omega_{1}\right), \boldsymbol{\Xi}\left(\theta_{2}, \omega_{2}\right), \ldots, \boldsymbol{\Xi}\left(\theta_{P}, \omega_{P}\right)\right]$, where

$$
\boldsymbol{\Xi}\left(\theta_{i}, \omega_{i}\right)=\mathbf{b}\left(\omega_{i}\right) \otimes \mathbf{a}\left(\theta_{i}\right)
$$

is the $M K \times 1$ spatiotemporal steering vector. This property is useful in the CRB analysis in Section 3.

Proof. See Appendix A. 


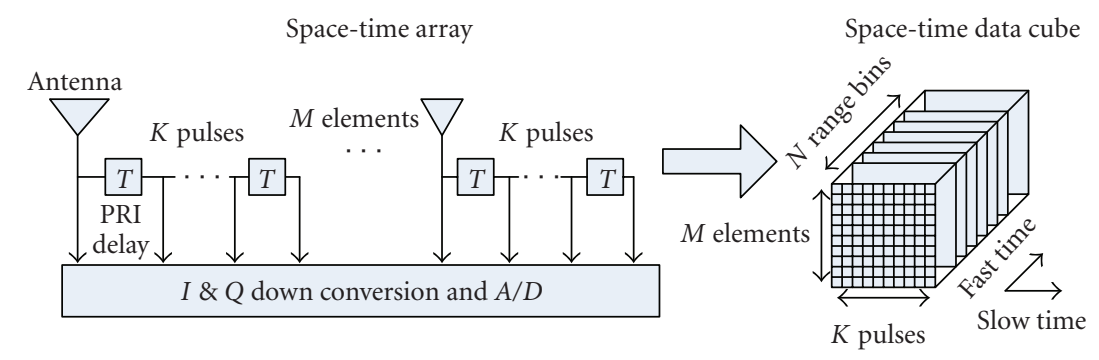

FIgURE 2: Space-time array radar data cube generation.

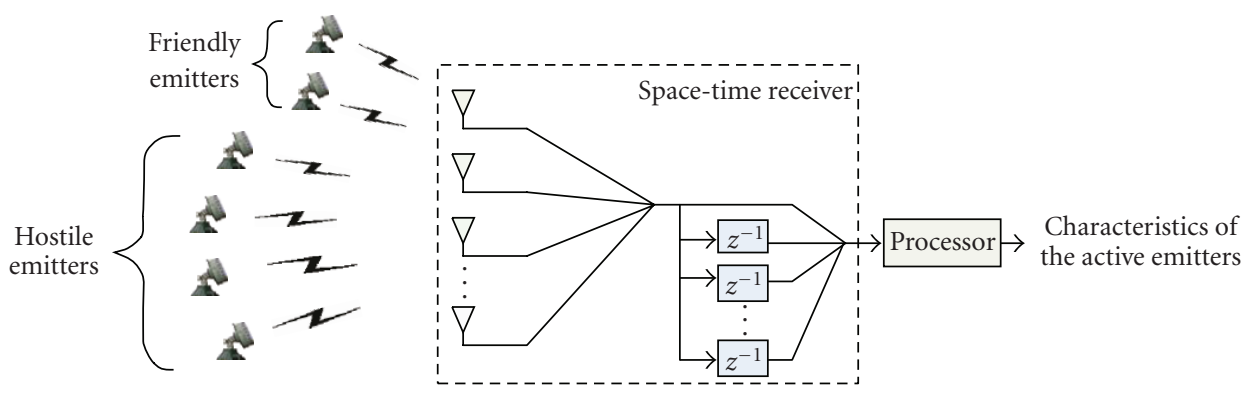

FIgURE 3: Space-time receiver architecture of the advanced ESM systems.

Property 2. With $K$-factor temporally smoothed data, up to $K$ sources having the same DOA, can be solved in this data model.

\section{Proof. See [5, Appendix A]}

Some assumptions associated with models (1) and (7) are as follows.

Assumption 1. The signals are unknown deterministic and uncorrelated with each other. Without loss of generality, we assume that $p_{1}(t)$ is the received waveform of the desired signal. We also assume that the transmitted waveform $p_{0}(t)$ of the desired signal is known a priori.

Assumption 2. The noise is circularly symmetric zero-mean Gaussian with variance $\sigma^{2}$. Both white noise and colored noise are considered in this paper. In the case of spatially and temporally white noise, the noise covariance matrix is $\mathbf{Q}=\sigma^{2} \mathbf{I}$, where $\mathbf{I}$ is the identity matrix.

Assumption 3. The number of sources $P$ is assumed to be known or has been estimated (see [25] on how to estimate the sources number $P$ from the input date $\mathbf{X}_{K}(t)$ ).

Assumption 4. $M K \geq P$, and the spatiotemporal manifold $\boldsymbol{\Omega}$ is unambiguous so that the spatiotemporal steering vectors $\Xi\left(\theta_{1}, \omega_{1}\right)$ and $\Xi\left(\theta_{2}, \omega_{2}\right)\left(\theta_{1} \neq \theta_{2}, \omega_{1} \neq \omega_{2}\right)$ are linearly independent. On the other hand, $M K$ is the upper bound on the number of sources that can be resolved in this data model whenever $\theta_{1} \neq \theta_{2}$ and $\omega_{1} \neq \omega_{2}$.

Assumption 5. Let $f_{s}$ be the sample rate, it is assumed that $f_{s}$ is large enough to the bandwidth of each narrowband source. To avoid aliasing, it is also required that $-f_{s} / 2<f_{i} \leq$ $f_{s} / 2$, where $f_{i}=f_{s} \omega_{i} /(2 \pi)$ is the baseband frequency before sampling.

\subsection{Some applications}

It is instructive to describe some applications where the data model and assumptions outlined above are relevant.

The first application where our data model and assumptions are reasonable is active array radar system [26]. In radar applications, a known waveform $p_{0}(t)$ is transmitted, and the received signal reflected from each target is just a scaled, time-delayed, and Doppler-shifted version of the transmitted signal. More specifically, consider a space-time array shown in Figure 2. The radar transmits a coherent train of $K$ pulses with the pulse repetition interval (PRI) $T$ in one coherent processing interval (CPI), and the target return collected by the space-time array with $M$ elements is an $M \times K \times N$ data cube, where $N$ is the number of snapshots (range bins). After I/Q down-conversion, each $M K \times 1$ snapshot vector has the form of (7).

The second application where our data model and assumptions hold true is the electronic support measures (ESM) signal processing $[27,28]$. The ESM systems perform the functions of threat detection and area surveillance. They use the passive antenna arrays to intercept the radar signal and determine the characteristics (e.g., radio frequency (RF), DOA, time of arrival (TOA), pulse width (PW), PRI, etc.) of the active emitters in a given area (see Figure 3 ). Moreover, advanced knowledge-based EMS systems also make full use of the priori information (e.g., the characteristics of friendly and enemy emitters) to enhance the performance. In this 


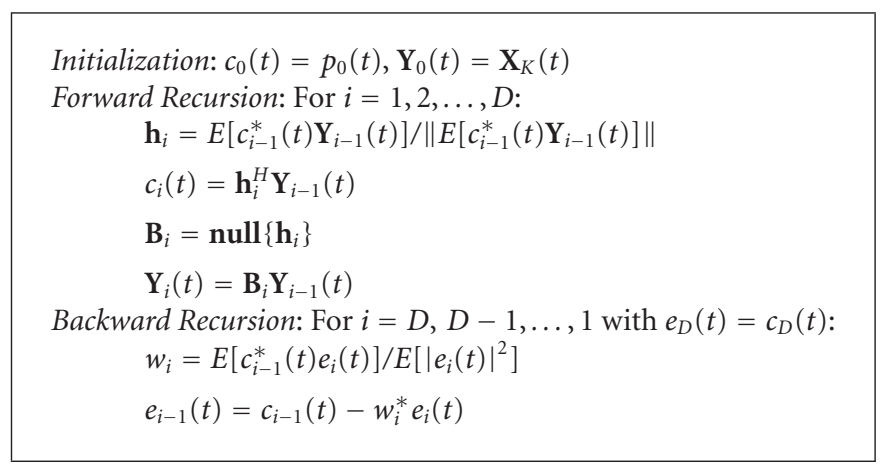

Algorithm 1: MWF algorithm [21].

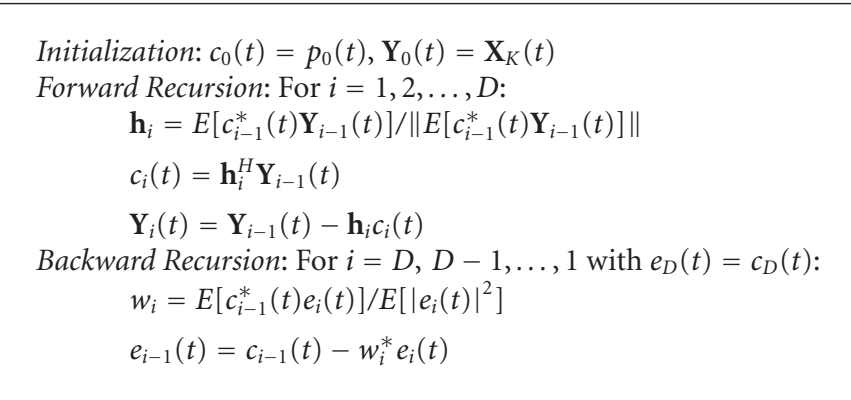

Algorithm 2: CSS-MWF algorithm [29].

application, the data stacking technique must be performed to create the spatiotemporal data.

\subsection{Multistage Wiener filter (MWF)}

In this section, we briefly review the MWF and its implementation using the correlation subtractive structure (CSS).

The MWF was developed by Goldstein et al. [21] based on orthogonal projections. A block diagram showing the structure of MWF is depicted in Figure 4. It is a multistage representation of the minimum mean-square error (MMSE) Wiener filer that generates a signal-dependent basis in a stage-by-stage structure. At every stage $i=1,2, \ldots, D$ of the decomposition, two orthogonal subspaces are formed: one in the direction of the $M K \times 1$ correlation vector $\mathbf{h}_{i}$, and the other orthogonal to $\mathbf{h}_{i}$. A blocking matrix $\mathbf{B}_{i}=\mathbf{n u l l}\left\{\mathbf{h}_{i}\right\}$ is also formed to perform the projection onto the subspace orthogonal to $\mathbf{h}_{i}$. It is clear that the scalar output $c_{i+1}(t)$ in the direction of $\mathbf{h}_{i}$ serves as the desired signal for the next stage while the vector output $\mathbf{Y}_{i+1}(t)$ orthogonal to $\mathbf{h}_{i}$ is the input vector of the next stage. The standard MWF algorithm is presented in Algorithm 1.

Note that the requirement for the blocking matrix $\mathbf{B}_{i}$ is

$$
\mathbf{B}_{i} \mathbf{h}_{i}=0 .
$$

Hence, the choice of $\mathbf{B}_{i}$ affects the computational complexity. To make the construction of $\mathbf{B}_{i}$ simple, an efficient imple- mentation of the MWF algorithm is proposed based on CSS [29]. First, the blocking matrix $\mathbf{B}_{i}$ is given by

$$
\mathbf{B}_{i}=\mathbf{I}-\mathbf{h}_{i} \mathbf{h}_{i}^{H} .
$$

Then, the input vector $\mathbf{Y}_{i}(t)$ for the $(i+1)$ th stage is calculated as follows:

$$
\mathbf{Y}_{i}(t)=\mathbf{B}_{i} \mathbf{Y}_{i-1}(t)=\left(\mathbf{I}-\mathbf{h}_{i} \mathbf{h}_{i}^{H}\right) \mathbf{Y}_{i-1}(t)=\mathbf{Y}_{i-1}(t)-\mathbf{h}_{i} c_{i}(t)
$$

The CSS-MWF algorithm is summarized in Algorithm 2.

From Algorithm 2, it is clear that CSS-MWF avoids the formation of blocking matrices, and thus, yields much lower computational complexity.

The MWF has the following properties.

(1) Let $\mathbf{T}_{D}=\left[\mathbf{h}_{1}, \mathbf{h}_{2}, \ldots, \mathbf{h}_{D}\right]$, where $D$ is the order of filter (in this paper, $D=M K$ ), it has been shown in $[21,23]$ that the columns in $\mathbf{T}_{D}$ are mutually orthogonal and each $\mathbf{h}_{i}(i=1,2, \ldots, D)$ is contained in the signal subspace.

(2) It is shown in [23] that the first $P$ orthogonal vectors span the signal subspace, and $P$ stages are required to form the full rank MMSE filter, where $P(P<D)$ is the number of sources. 


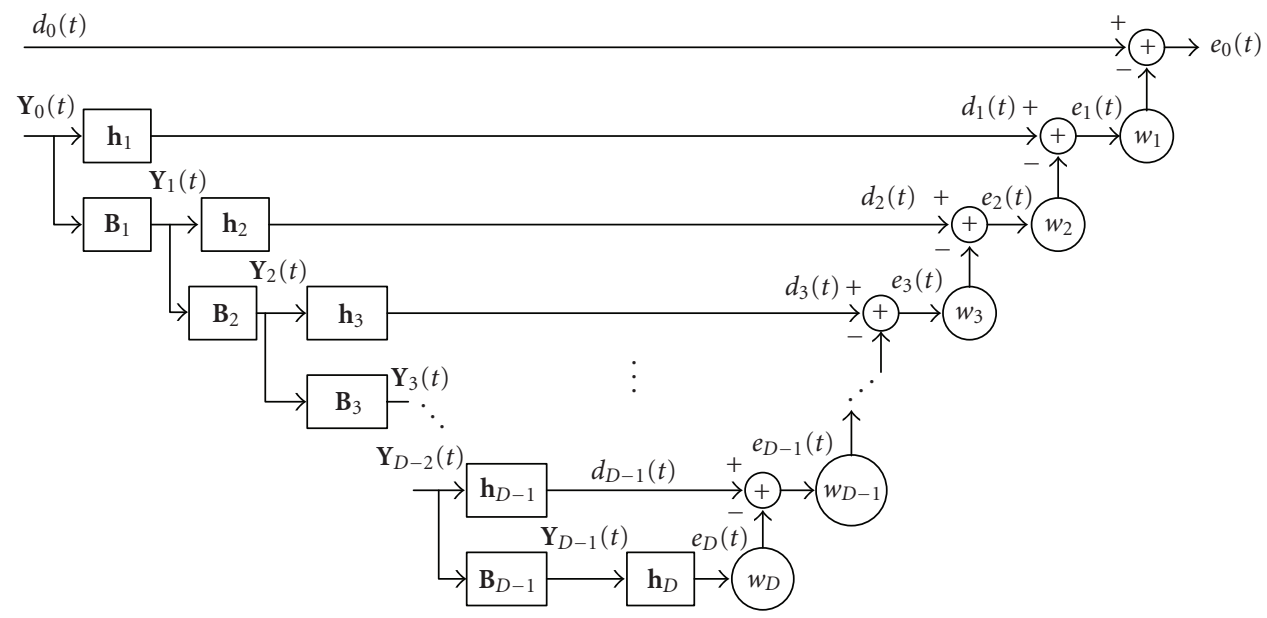

Figure 4: Multistage Wiener filter.

\subsection{Proposed method}

Let $\widehat{\mathbf{\Omega}}=\left[\mathbf{h}_{1}, \mathbf{h}_{2}, \ldots, \mathbf{h}_{P}\right]$ denote the matrix of the first $P$ basis vectors of the MWF. In the case of high signal-to-noise ratio (SNR) or large snapshots number $N$, we have

$$
\widehat{\mathbf{\Omega}} \approx \mathbf{\Omega} \mathbf{H}
$$

where $\mathbf{H}$ is a $P \times P$ nonsingular matrix. Moreover, $\hat{\boldsymbol{\Omega}}$ is consistent in the sense that $\lim _{N \rightarrow \infty} \hat{\boldsymbol{\Omega}}=\mathbf{\Omega} \mathbf{H}$. This implies that the corresponding transformed matrices for $\mathbf{A}$ and $\boldsymbol{\Phi}$ can be expressed as

$$
\begin{gathered}
\mathbf{A}_{T}=\mathbf{A} \mathbf{H}, \\
\boldsymbol{\Phi}_{T}=\mathbf{H}^{-1} \boldsymbol{\Phi} \mathbf{H},
\end{gathered}
$$

and they can be estimated as follows:

$$
\widehat{\mathbf{A}}_{T}=\hat{\mathbf{\Omega}}_{1: 1}, \quad \hat{\boldsymbol{\Phi}}_{T}=\hat{\boldsymbol{\Omega}}_{1: K-1}^{\#} \hat{\mathbf{\Omega}}_{2: K},
$$

where $\hat{\boldsymbol{\Omega}}_{k: l}$ denotes the block rows from $k$ through $l$.

Since (14) is a similarity transformation, $\boldsymbol{\Phi}_{T}$ and $\boldsymbol{\Phi}$ have the same eigenvalues $e^{j \omega_{i}}(i=1,2, \ldots, P)$ in the noise-free case. By performing the eigendecomposition $\hat{\boldsymbol{\Phi}}_{T}=\mathbf{U} \boldsymbol{\Lambda} \mathbf{U}^{-1}$ $\left(\boldsymbol{\Lambda}=\operatorname{diag}\left[\xi_{1}, \xi_{2}, \ldots, \xi_{P}\right]\right)$, we obtain the eigenvalues of $\hat{\boldsymbol{\Phi}}_{T}$, namely, $\xi_{i}(i=1,2, \ldots, P)$. Therefore, the frequency estimates are given by

$$
\widehat{\omega}_{i}=\angle \xi_{i}, \quad i=1,2, \ldots, P .
$$

On the other hand, since $\mathbf{U}$ diagonalizes $\widehat{\boldsymbol{\Phi}}_{T}$, it provides an estimation of $\mathbf{H}^{-1}$ in (14). Therefore, the steering matrix A can be estimated as $\widehat{\mathbf{A}}=\widehat{\mathbf{A}}_{T} \mathbf{U}$. Letting $\hat{\mathbf{a}}_{i}$ denote the $i$ th column of $\widehat{\mathbf{A}}$, for large $N$, we have $\hat{\mathbf{a}}_{i} \propto \mathbf{a}\left(\theta_{i}\right)$. Since the steering matrix A for the ULA is a Vandermonde matrix, in the noise-free case, we obtain

$$
\begin{gathered}
\frac{\hat{\mathbf{a}}_{i}(2)}{\hat{\mathbf{a}}_{i}(1)}=\frac{\hat{\mathbf{a}}_{i}(3)}{\hat{\mathbf{a}}_{i}(2)}=\cdots=\frac{\hat{\mathbf{a}}_{i}(M)}{\hat{\mathbf{a}}_{i}(M-1)}=e^{j 2 \pi\left(d \sin \hat{\theta}_{i} / \lambda_{i}\right)}, \\
i=1,2, \ldots, P .
\end{gathered}
$$

Then, we can derive the DOA estimates from (17) as

$$
\hat{\theta}_{i}=\frac{1}{M-1} \sum_{l=2}^{M} \sin ^{-1}\left(\frac{\hat{\lambda}_{i}}{2 \pi d} \angle\left(\frac{\hat{\mathbf{a}}_{i}(l)}{\hat{\mathbf{a}}_{i}(l-1)}\right)\right), \quad i=1,2, \ldots, P,
$$

where $\hat{\lambda}_{i}$ can be calculated by using the frequency estimates $\widehat{\omega}_{i}$ in (16) and the center frequency of the band of interest $\omega_{c}$.

The idea of DOA estimation is similar to the method of [6] (referred to as the Viberg-Stoica method) which avoids the operation of joint diagonalization in $[4,5]$, but we give the closed form of DOA estimates. From (16) and (18), it is clear that $\hat{\omega}_{i}$ and $\hat{\theta}_{i}$ are one-to-one related to the $i$ th eigenvalue and the $i$ th eigenvector, respectively. In other words, the frequency and DOA estimates are paired automatically.

The proposed method is summarized in the following steps.

S1: Estimate the signal subspace $\hat{\boldsymbol{\Omega}}$ by performing the forward recursion of the $\operatorname{rank} P$ MWF, where $P$ is the number of sources.

S2: Estimate the transformed matrices for $\mathbf{A}$ and $\boldsymbol{\Phi}$ from (15).

S3: Perform the eigendecomposition $\widehat{\boldsymbol{\Phi}}_{T}=\mathbf{U} \boldsymbol{\Lambda} \mathbf{U}^{-1}$, and obtain the eigenvalues of $\hat{\boldsymbol{\Phi}}_{T}$. Then, estimate the frequencies from (16).

S4: Estimate the steering matrix $\mathbf{A}$ as $\widehat{\mathbf{A}}=\widehat{\mathbf{A}}_{T} \mathbf{U}$. Then, the DOAs can be estimated from (18). 
After obtaining the estimates of DOA/frequency pairs $\left(\hat{\theta}_{i}, \hat{\omega}_{i}\right)(i=1,2, \ldots, P)$, we can use the known transmitted waveform $p_{0}(t)$ to extract the desired signal DOA/frequency pair by using the cross correlation method in [13].

\section{Remarks}

(1) In STAP airborne radar application, it is shown in [22] that the MWF cannot only achieve a substantially higher compression of the interference subspace than the classical subspace-based techniques (e.g., principle components (PC) method and crossspectral metric (CSM) method) in both hot and cold clutter environment, but also provide robustness to eigenspectrum spreading or subspace leakage of the interference subspace. Thus, it has the potential for making the proposed method more feasible in the presence of colored noise.

(2) It is very important to notice that the CSS-MWF algorithm only involves complex matrix-vector products, and requires the computationally complexity of $O(M K N)$ floating-point operations per second (flops) at each stage [29]. Therefore, the complexity of $O(P M K N)$ flops is required to estimate the signal subspace $\widehat{\boldsymbol{\Omega}}$ of rank $P$ by performing the forward recursion of the MWF. In contrast to the classical subspace-based methods of $[3,4,6]$ which require $O\left((M K)^{2} N\right)+O\left(M^{3} K^{3}\right)$ flops in estimating the covariance matrix and calculating the eigendecomposition, the proposed method shows lowcomplexity capability.

\subsection{Cramér-Rao bound}

Although the complete statistical analysis of the estimation algorithm is not the scope of this paper, it is still useful to present the CRB that indicates the performance limit of any unbiased estimator.

In the literature, a large number of researchers have studied the conditional and unconditional CRB for DOA estimation (see, e.g., [30-33] and the references therein). In this section, we derive the expression of the CRB for joint DOA and frequency estimation. The new expressions of CRB can be viewed as an extension of the well-known results of Stoica and Nehorai [30]. Since the signals are assumed to be unknown deterministic, we only consider the conditional CRB.

For simplicity, we rewrite the data model (7) as

$$
\mathbf{X}_{K}(t)=\mathbf{\Omega} \mathbf{g}(t)+\mathbf{N}_{K}(t), \quad t=0,1, \ldots, N-1,
$$

where $\mathbf{g}(t)=\boldsymbol{\Phi}^{t} \mathbf{s}(t)=\left[\begin{array}{llll}g_{1}(t) & g_{2}(t) & \cdots & g_{P}(t)\end{array}\right]^{T}$.

Theorem 1. Under the assumptions in Section 2.1, the conditional CRB for joint DOA and frequency estimation in white noise can be expressed as

$$
\mathbf{C R B}(\boldsymbol{\theta}, \boldsymbol{\omega})=\frac{\sigma^{2}}{2}\left[\sum_{t=1}^{N} \operatorname{Re}\left\{\mathbf{Z}^{H}(t) \mathbf{D}^{H} \mathbf{P}_{\mathbf{\Omega}}^{\perp} \mathbf{D Z}(t)\right\}\right]^{-1},
$$

where

$$
\begin{aligned}
& \mathbf{Z}(t)=\left[\begin{array}{cc}
\mathbf{G}(t) & 0 \\
0 & \mathbf{G}(t)
\end{array}\right], \\
& \mathbf{G}(t)=\operatorname{diag}\left\{\begin{array}{llll}
g_{1}(t) & g_{2}(t) & \cdots & g_{P}(t)
\end{array}\right\}, \\
& \mathbf{D}=\left[\begin{array}{ll}
\mathbf{D}_{\theta} & \mathbf{D}_{\omega}
\end{array}\right], \\
& \mathbf{D}_{\boldsymbol{\theta}}=\left[\begin{array}{llll}
d_{\boldsymbol{\theta}}\left(\theta_{1}, \omega_{1}\right) & d_{\boldsymbol{\theta}}\left(\theta_{2}, \omega_{2}\right) & \cdots & d_{\boldsymbol{\theta}}\left(\theta_{P}, \omega_{P}\right)
\end{array}\right], \\
& \mathbf{D}_{\boldsymbol{\omega}}=\left[\begin{array}{llll}
d_{\boldsymbol{\omega}}\left(\theta_{1}, \omega_{1}\right) & d_{\boldsymbol{\omega}}\left(\theta_{2}, \omega_{2}\right) & \cdots & d_{\boldsymbol{\omega}}\left(\theta_{P}, \omega_{P}\right)
\end{array}\right], \\
& d_{\theta}\left(\theta_{i}, \omega_{i}\right)=\left.\frac{\partial \Xi(\theta, \omega)}{\partial \theta}\right|_{\theta=\theta_{i}, \omega=\omega_{i}}, \\
& d_{\omega}\left(\theta_{i}, \omega_{i}\right)=\left.\frac{\partial \Xi(\theta, \omega)}{\partial \omega}\right|_{\theta=\theta_{i}, \omega=\omega_{i}}, \\
& \mathbf{P}_{\boldsymbol{\Omega}}^{\perp}=\mathbf{I}-\boldsymbol{\Omega}\left(\boldsymbol{\Omega}^{H} \boldsymbol{\Omega}\right)^{-1} \boldsymbol{\Omega}^{H} .
\end{aligned}
$$

Proof. See Appendix B.

Theorem 2. For large $N$, the asymptotic conditional CRB for joint DOA and frequency estimation in white noise can be expressed as

$$
\mathbf{C R B}(\boldsymbol{\theta}, \boldsymbol{\omega}) \approx \frac{\sigma^{2}}{2 N}\left[\operatorname{Re}\left\{\left[\mathbf{D}^{H} \mathbf{P}_{\Omega}^{\perp} \mathbf{D}\right] \odot \mathfrak{R}^{T}\right\}\right]^{-1},
$$

where

$$
\mathfrak{R}=\left[\begin{array}{ll}
\mathbf{R}_{\mathbf{g}} & \mathbf{R}_{\mathbf{g}} \\
\mathbf{R}_{\mathbf{g}} & \mathbf{R}_{\mathbf{g}}
\end{array}\right], \quad \mathbf{R}_{\mathbf{g}}=\lim _{N \rightarrow \infty} \frac{1}{N} \sum_{t=1}^{N} \mathbf{g}(t) \mathbf{g}^{H}(t) .
$$

Proof. See Appendix C.

The asymptotic CRB for DOA estimation in the colored noise is derived in [34]. By extending the results of [34], we may obtain the expression of the condition CRB for joint DOA and frequency estimation in the colored noise.

Theorem 3. The asymptotic conditional CRB for joint DOA and frequency estimation in colored noise can be expressed as

$$
\mathbf{C R B}(\boldsymbol{\theta}, \boldsymbol{\omega}) \approx \frac{\sigma^{2}}{2 N}\left[\operatorname{Re}\left\{\left[\mathbf{D}^{H} \mathbf{Q}^{-1} \mathbf{P}_{\tilde{\mathbf{\Omega}}}^{\perp} \mathbf{D}\right] \odot \mathfrak{R}^{T}\right\}\right]^{-1},
$$

where $\mathbf{P}_{\widetilde{\mathbf{\Omega}}}^{\perp}=\mathbf{I}-\boldsymbol{\Omega}\left(\boldsymbol{\Omega}^{H} \mathbf{Q}^{-1} \boldsymbol{\Omega}\right)^{-1} \boldsymbol{\Omega}^{H} \mathbf{Q}^{-1}$. The noise covariance matrix $\mathbf{Q}$ is no longer a diagonal matrix in the case of colored noise.

\section{SIMULATION AND EXPERIMENTAL RESULTS}

In this section, we present simulation and experimental examples showing the performance of the proposed method. The situation in which there is one desired signal with known transmitted waveform $p_{0}(t)$ in the presence of interfering signals is considered. 
TABLE 1: Comparisons of the computational complexity of various algorithms.

\begin{tabular}{ll}
\hline Algorithms & Main computational complexity \\
\hline JAFE & High-dimensional SVD: $O\left((M K)^{2} N\right)+O\left(M^{3} K^{3}\right)+$ two low dimensional EVD: $O\left(P^{3}\right)$ \\
Viberg-Stoica method & High-dimensional SVD: $O\left((M K)^{2} N\right)+O\left(M^{3} K^{3}\right)+$ low dimensional EVD: $O\left(P^{3}\right)$ \\
FSF-MUSIC & Three low-dimensional SVDs: $2 O\left(K^{2} N\right)+2 O\left(K^{3}\right)+O\left(M^{2} N\right)+O\left(M^{3}\right)+$ three 1 -D searches \\
Proposed method & Forward recursions of the CSS-MWF: $O(P M K N)+$ low dimensional EVD: $O\left(P^{3}\right)$ \\
\hline
\end{tabular}

\subsection{Simulation examples}

In the simulation examples below, the array is assumed to be a ULA with interelement spacing equal to a half wavelength $\left(\lambda=2 \pi c / \omega_{c}\right)$.

Example 1. In this example, we assume that there are three uncorrelated narrowband sources with equal power impinging on the array from far filed. The number of sensors is $M=6$, the temporal smoothing factor is $K=2$, and the number of snapshots is $N=100$. The DOA/Frequency pairs of the three sources are $\left(5^{\circ}, 1.6 \mathrm{rad}\right),\left(-5^{\circ}, 1.9 \mathrm{rad}\right)$, and $\left.5^{\circ}, 2.2 \mathrm{rad}\right)$, respectively. Figure 5 shows the scatter plots of proposed method at SNRs $=10 \mathrm{~dB}$. We observe that the resulting estimates are paired automatically. Moreover, we note that the two sources with the same DOAs $=5^{\circ}$ are clearly resolved. This is consistent with Property 2 in Section 2.1.

Example 2. This example evaluates the performance of proposed method for different angle and frequency separations. We assume that the number of sensors is $M=8$, and the number of snapshots is $N=100$. Thus, the Fourier temporal resolution limit is $2 \pi / N$ rad or $0.0628 \mathrm{rad}$ and the Rayleigh angle resolution limit for the ULA is $2 /(M-1) \mathrm{rad}$ or $16.38^{\circ}$. First, it is assumed that two sources come from $\theta_{1}=0^{\circ}$ and $\theta_{2}=(0+\Delta \theta)^{\circ}$ with two different frequencies $\omega_{1}=$ $2.1 \mathrm{rad}$ and $\omega_{2}=2.5 \mathrm{rad}$, respectively, where $\Delta \theta$ is the angle separation between the sources. Figures 6(a) and 6(b) show the root-mean-square errors (RMSEs) of $\hat{\omega}_{1}$ and $\hat{\theta}_{1}$ versus angle separation $\Delta \theta$ at SNRs $=15 \mathrm{~dB}$. The performance of the second source is similar to that of the first one. All results provided contain 1000 Monte Carlo trials. The RMSEs of the $i$ th source for DOA and frequency estimation are, respectively, defined as

$$
\begin{gathered}
\operatorname{RMSEs}_{\hat{\theta}_{i}}=\sqrt{E\left[\left(\hat{\theta}_{i}-\theta_{i}\right)^{2}\right]}, \quad i=1,2, \ldots, P, \\
\operatorname{RMSEs}_{\hat{\omega}_{i}}=\sqrt{E\left[\left(\hat{\omega}_{i}-\omega_{i}\right)^{2}\right]}, \quad i=1,2, \ldots, P,
\end{gathered}
$$

where $i$ represents the source index. For a clear illustration, only the square root of the CRB (RCRB) with $K=4$ is provided. Figures $6(\mathrm{a})$ and $6(\mathrm{~b})$ show that, as the temporal smoothing factor $K$ increases, the accuracy is improved. We also note from Figures 6(a) and 6(b) that the two sources with the same DOA (when $\Delta \theta=0$ ) can be resolved by using the spatiotemporal data model, which is again consistent with the discussion of Property 2 in Section 2.1 .

Then, we assume that two sources with the frequencies $\omega_{1}=2.1 \mathrm{rad}$ and $\omega_{2}=(2.1+\Delta \omega) \mathrm{rad}$ come from two

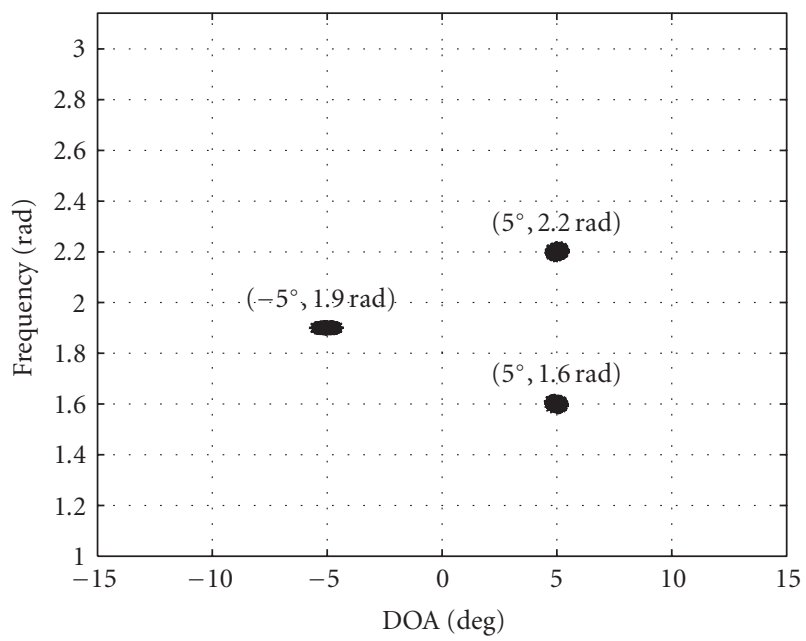

FIGURE 5: Scatter plot of estimated DOA/frequency pairs with proposed method. SNRs $=10 \mathrm{~dB}, M=6, K=2, N=100$, and 1000 trials are used.

different DOAs $\theta_{1}=5^{\circ}$ and $\theta_{2}=10^{\circ}$, respectively, where $\Delta \omega$ is the frequency separation between the sources. Figures 7 (a) and 7(b) show the RMSEs of $\hat{\omega}_{1}$ and $\hat{\theta}_{1}$ versus frequency separation $\Delta \omega$ at SNRs $=15 \mathrm{~dB}$. We observe once again that the temporal smoothing can improve the accuracy. However, unlike the results in Figures 6(a) and 6(b), two sources with the same frequency (when $\Delta \omega=0$ ) cannot be resolved by using the spatiotemporal data model. Meanwhile, Figures 7 (a) and 7(b) show that the temporal resolution of the proposed method goes beyond its corresponding resolution limit. Moreover, it is seen that, as the frequency separation $\Delta \omega$ increases, the accuracy of DOA estimation is improved while the improvement for frequency estimation is little.

Example 3. This example tests the RMSEs of proposed method versus the SNR in both white noise and colored noise. Comparisons with the JAFE algorithm [4], the VibergStoica method [6], the FSF-MUSIC algorithm [9], and the RCRB are made simultaneously. In the simulations below, the number of sources is $P=2$. The true DOA/Frequency pairs of the two sources are $\left(-3^{\circ}, 2.1 \mathrm{rad}\right)$ and $\left(3^{\circ}, 2.15 \mathrm{rad}\right)$, respectively. The number of sensors is $M=12$, the temporal smoothing factor is $K=4$, and the number of snapshots is $N=100$. Thus, both the temporal resolution and the spatial resolution of the proposed method go beyond their corresponding resolution limits $\left(0.0628 \mathrm{rad}\right.$ and $\left.10.42^{\circ}\right)$. All results provided contain 1000 Monte Carlo trials. 
TABLe 2: Means and RMSEs of three methods based on the 20 estimates when used with experimental data.

\begin{tabular}{lcccccc}
\hline \multirow{2}{*}{ Source $\left(\theta_{i}, f_{i}\right)$} & \multicolumn{2}{c}{ Prewhitened JAFE } & \multicolumn{2}{c}{ Method of Zoltowski } & \multicolumn{2}{c}{ Proposed method } \\
& Mean & RMSE & Mean & RMSE & Mean & RMSE \\
\hline$\left(3^{\circ}, 0.3436\right)$ & $\left(3.052^{\circ}, 0.3409\right)$ & $\left(0.3162^{\circ}, 0.002613\right)$ & $\left(2.879^{\circ}, 0.3396\right)$ & $\left(0.8163^{\circ}, 0.003921\right)$ & $\left(3.056^{\circ}, 0.3429\right)$ & $\left(0.2899^{\circ}, 0.002139\right)$ \\
$\left(-9^{\circ}, 0.25\right)$ & $\left(-9.051^{\circ}, 0.2532\right)$ & $\left(0.2854^{\circ}, 0.002159\right)$ & $\left(-8.810^{\circ}, 0.2558\right)$ & $\left(0.8631^{\circ}, 0.004051\right)$ & $\left(-9.033^{\circ}, 0.2520\right)$ & $\left(0.3484^{\circ}, 0.002386\right)$ \\
$\left(8^{\circ}, 0.1563\right)$ & $\left(8.074^{\circ}, 0.1571\right)$ & $\left(0.3509^{\circ}, 0.002273\right)$ & $\left(8.261^{\circ}, 0.1611\right)$ & $\left(0.7972^{\circ}, 0.004237\right)$ & $\left(8.062^{\circ}, 0.1557\right)$ & $\left(0.3737^{\circ}, 0.002751\right)$ \\
\hline
\end{tabular}

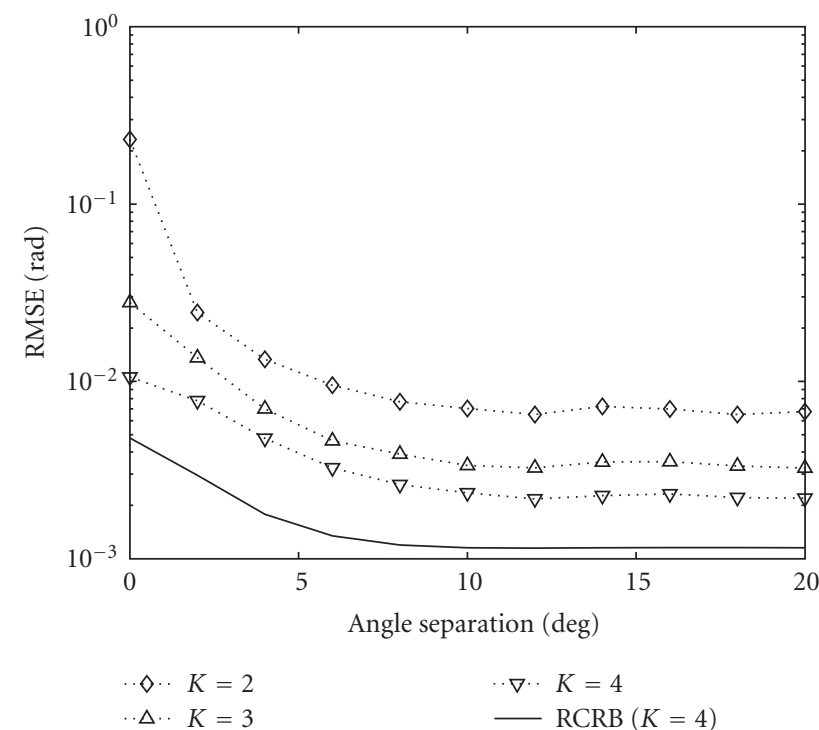

(a)

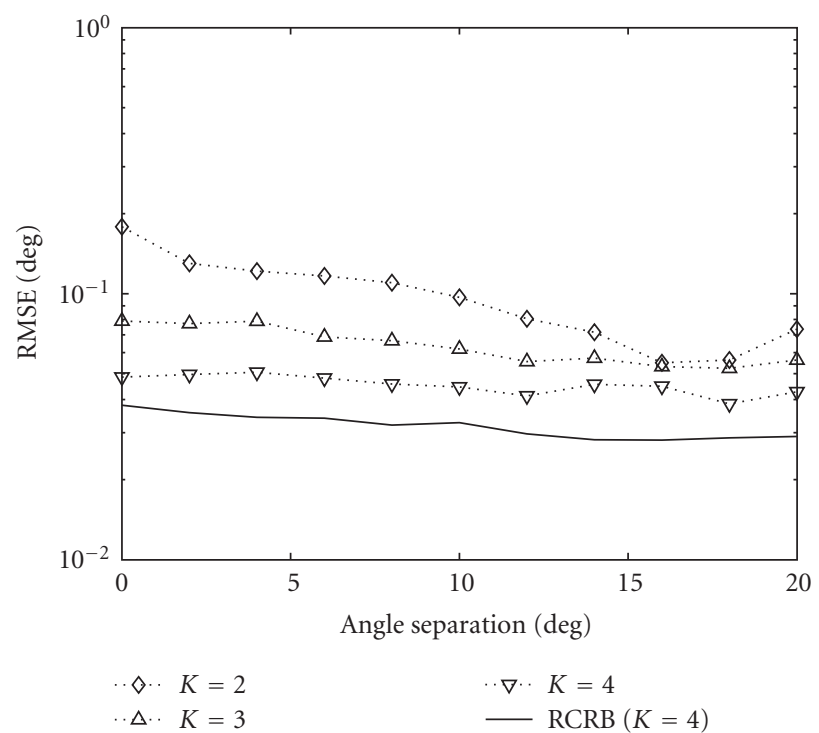

(b)

FIGURE 6: RMSE curves of the proposed method for frequency and DOA estimation of the first signal versus angle separation with fixed SNRs $=15 \mathrm{~dB}, M=8$, and $N=100$. (a) Frequency estimation. (b) DOA estimation.

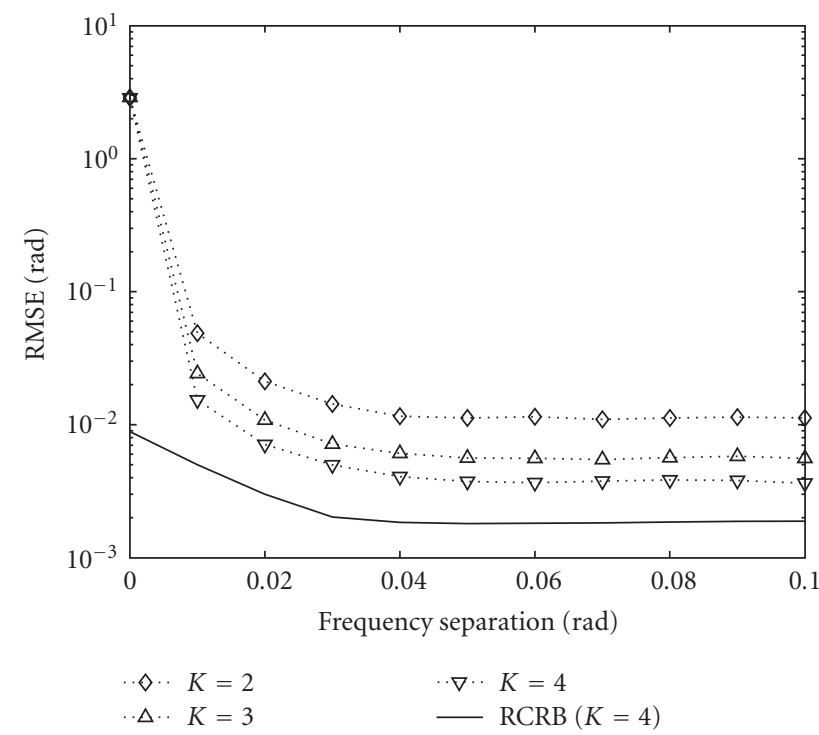

(a)

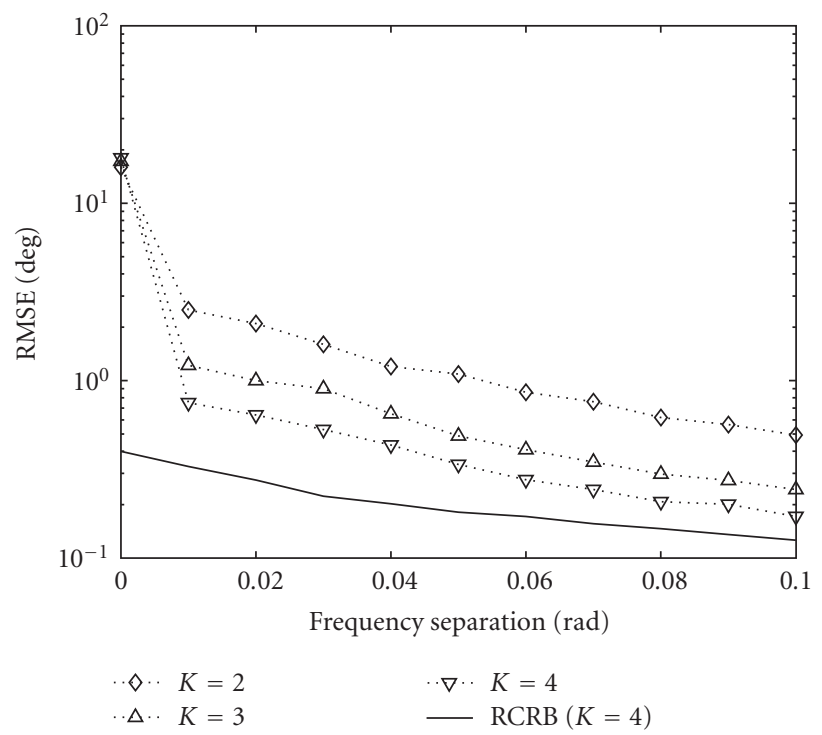

(b)

FIGURE 7: RMSE curves of the proposed method for frequency and DOA estimation of the first signal versus frequency separation with fixed SNRs $=15 \mathrm{~dB}, M=8$, and $N=100$. (a) Frequency estimation. (b) DOA estimation. 


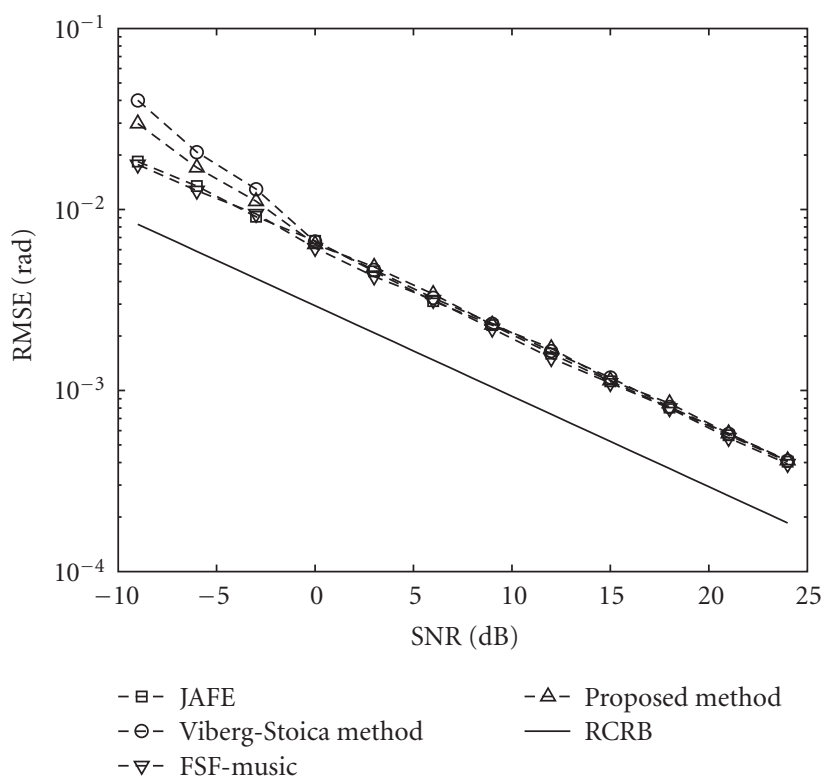

(a)

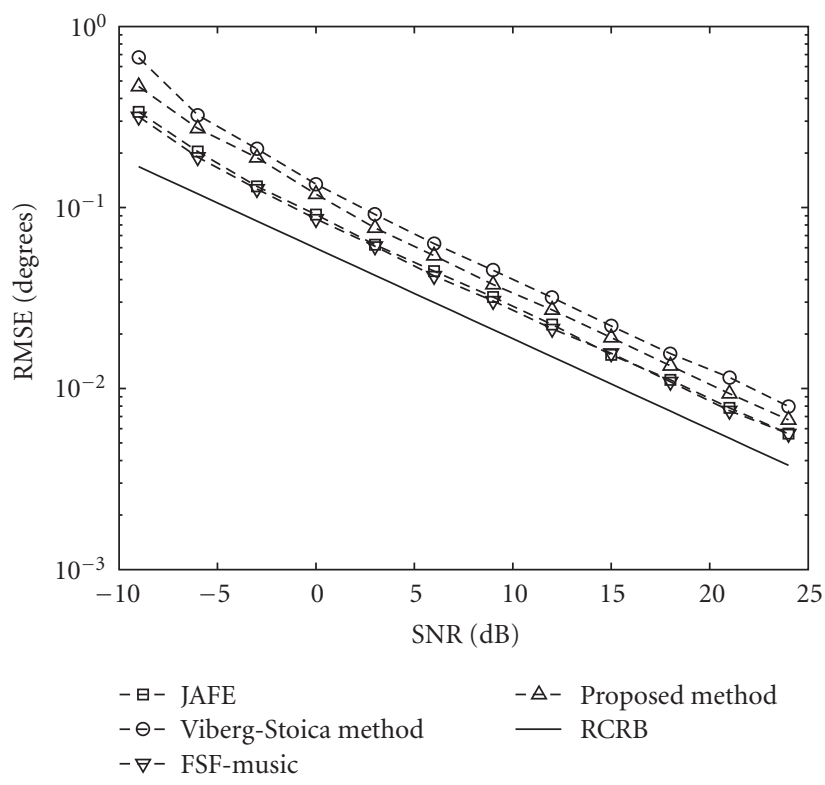

(b)

FIGURE 8: RMSE curves of four methods for frequency and DOA estimation of the first signal versus SNR and the corresponding RCRB in both spatially and temporally white noise with fixed $M=12, N=100$, and $K=4$. (a) Frequency estimation. (b) DOA estimation.

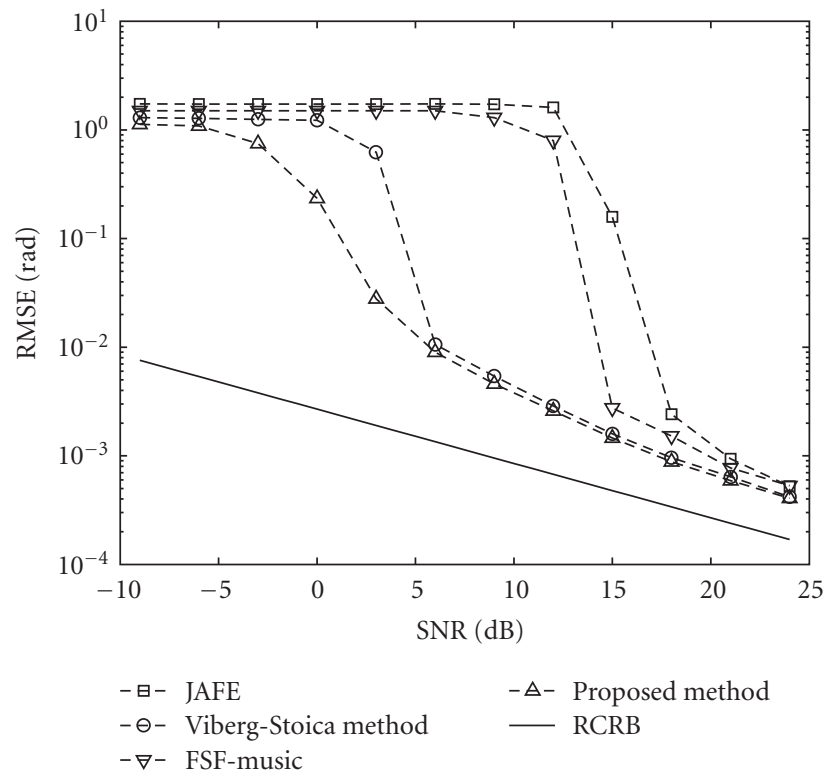

(a)

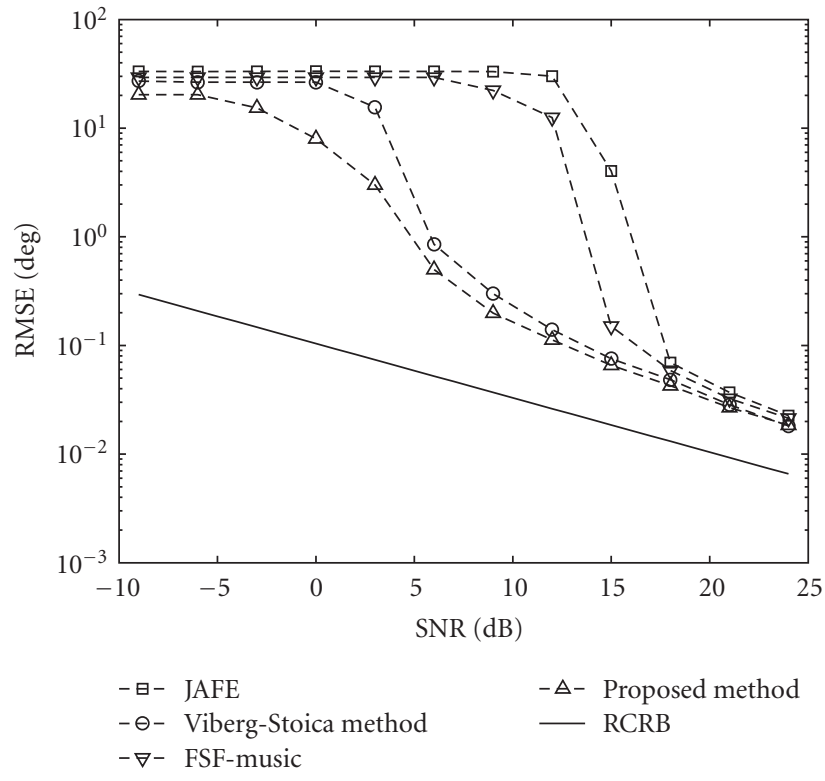

(b)

FIGURE 9: RMSE curves of four methods for frequency and DOA estimation of the first signal versus SNR and the corresponding RCRB in both spatially and temporally colored noise with fixed $M=12, N=100$, and $K=4$. (a) Frequency estimation. (b) DOA estimation.

First, we assume that the noise is both spatially and temporally white. Figures $8(\mathrm{a})$ and $8(\mathrm{~b})$ show the RMSE curves of frequency and DOA estimates versus SNR for the first source. The performance of the second source is similar to that of the first one. From Figures 8(a) and 8(b), it is obvious that the JAFE and the FSF-MUSIC have very close performances and outperform other two methods for both frequency and DOA estimations. Meanwhile, the performance of the proposed method is slightly superior to that of the Viberg-Stoica method.

Then, we consider a more general scenario where the noise is both spatially and temporally colored. Figures 9(a) and 9(b) show the RMSE curves versus SNR for the first signal in the colored noise which is modeled as a multichannel 


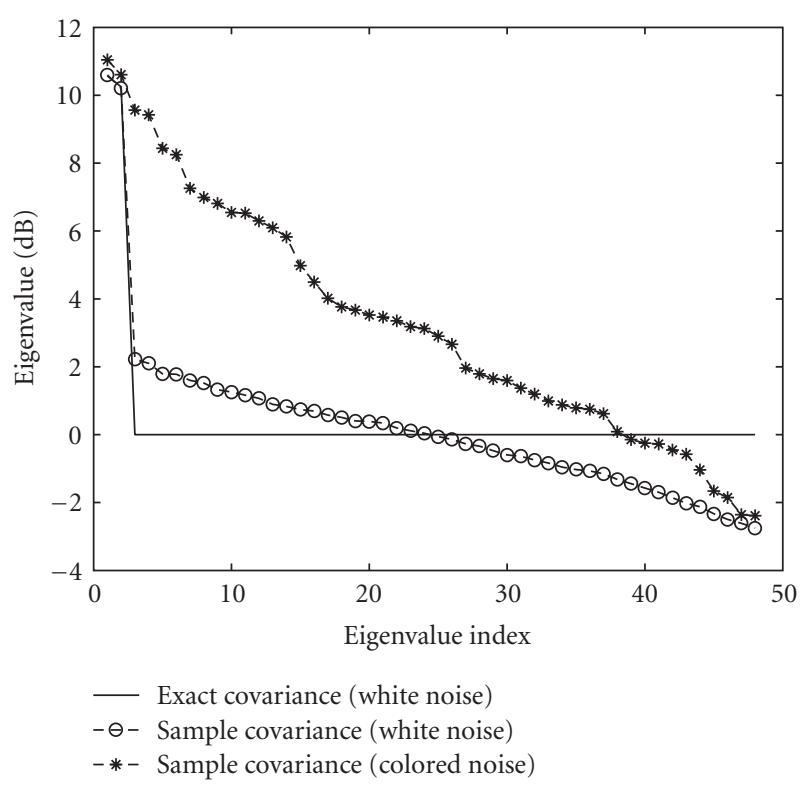

FIGURE 10: Eigenvalue spectrum of the sample covariance matrix with $M=12, K=4, N=100$, and SNRs $=10 \mathrm{~dB}$.

second-order autoregressive (AR(2)) random process [35]. Note that the proposed method has the best performance among the four methods for both frequency and DOA estimations, especially in the low SNR region. To gain insight into why the colored noise degrades the performances of the classical subspace-based methods significantly, we plot the eigenvalue spectrum of aforementioned simulation examples in Figure 10 for both white noise case and colored noise case. It is clear that the presence of colored noise leads to the eigenspectrum spreading. In this situation, the noise subspace is not orthogonal to the signal subspace anymore. Moreover, we give another important measure of performance in this analysis, namely, the subspace distance [36]. The subspace distance is a measure that compares the Euclidian distance between two subspaces. The smaller the subspace distance is, the more similar the two subspaces are. Let $\hat{\boldsymbol{\Omega}}_{\mathrm{MWF}}$ and $\hat{\boldsymbol{\Omega}}_{\mathrm{EIG}}$ denote two signal subspace estimates based on MWF and eigendecomposition, respectively. The signal subspace distances between $\hat{\boldsymbol{\Omega}}_{\mathrm{MWF}}$ (or $\hat{\boldsymbol{\Omega}}_{\mathrm{EIG}}$ ) and the true signal subspace $\boldsymbol{\Omega}$ are defined as follows [36]:

$$
d_{\hat{\mathbf{\Omega}}_{\mathrm{MWF}}}=\frac{1}{\sqrt{2}}\left\|\mathbf{P}_{\hat{\mathbf{\Omega}}_{\mathrm{MWF}}}-\mathbf{P}_{\boldsymbol{\Omega}}\right\|_{F}, \quad d_{\hat{\mathbf{\Omega}}_{\mathrm{EIG}}}=\left.\frac{1}{\sqrt{2}}\left\|\mathbf{P}_{\hat{\mathbf{\Omega}}_{\mathrm{EIG}}}-\mathbf{P}_{\boldsymbol{\Omega}}\right\|\right|_{F},
$$

where $\mathbf{P}_{\hat{\mathbf{\Omega}}_{\mathrm{MWF}}}, \mathbf{P}_{\hat{\mathbf{\Omega}}_{\mathrm{EIG}}}$, and $\mathbf{P}_{\boldsymbol{\Omega}}$ are three orthogonal projection matrices onto the spaces of $\hat{\boldsymbol{\Omega}}_{\mathrm{MWF}}, \hat{\boldsymbol{\Omega}}_{\mathrm{EIG}}$, and $\boldsymbol{\Omega}$, respectively. Figures 11(a) and 11(b) show the comparisons of the signal subspace distances $d_{\hat{\Omega}_{\mathrm{MWF}}}$ and $d_{\hat{\mathbf{\Omega}}_{\mathrm{EIG}}}$ for the same simulation scenario as Figures 8 and 9. We can note from Figure 11(a) that the subspace distances $d_{\hat{\Omega}_{\mathrm{MWF}}}$ and $d_{\hat{\Omega}_{\mathrm{EIG}}}$ are very close in the case of white noise. However, the subspace distance $d_{\hat{\Omega}_{\mathrm{MWF}}}$ is significantly smaller than the distance $d_{\hat{\Omega}_{\mathrm{EIG}}}$ in Figure $11(\mathrm{~b})$ especially in the low SNR region. In this case, the subspace determined by $\hat{\boldsymbol{\Omega}}_{\text {EIG }}$ departs from the true signal subspace $\boldsymbol{\Omega}$, which results in a drastic performance degradation.

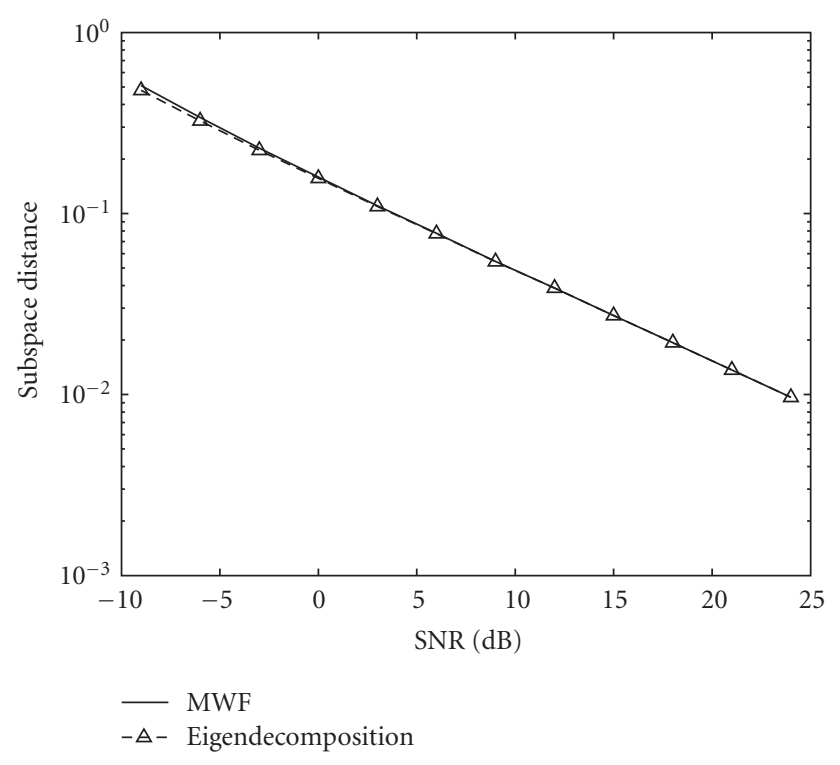

(a)

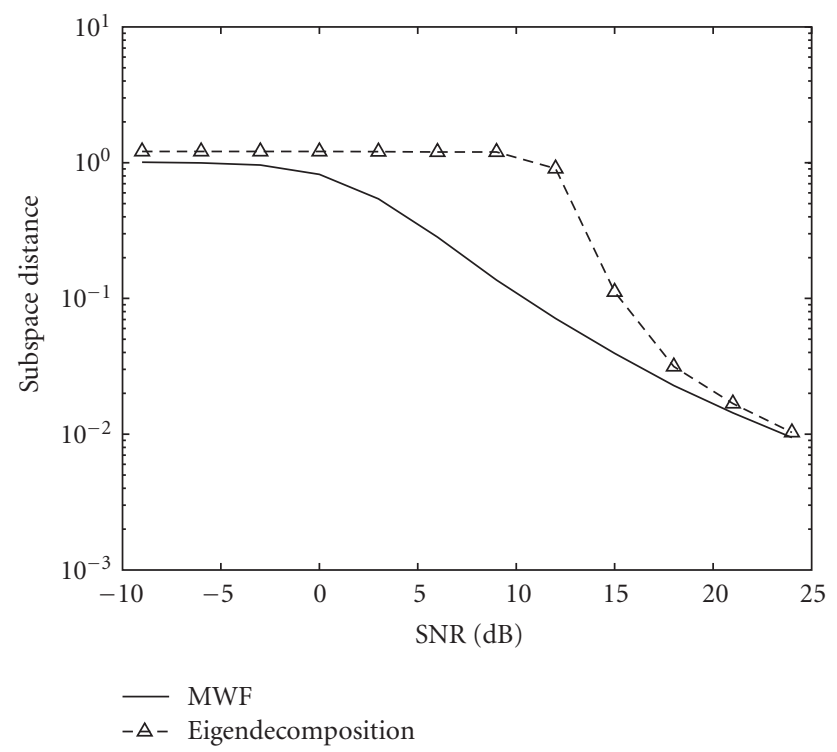

(b)

Figure 11: Comparison of the signal subspace distances with fixed $M=12, N=100$, and $K=4$. (a) White noise. (b) Colored noise.

\section{Comments}

The classical subspace-based methods are inherently not well suited to the situation in which a dominant signal subspace is not clearly present. In addition, it should be noted that, although the prewhitened subspace-based method [6] can improve the accuracy, known statistics of the colored noise (e.g., the temporal and spatial correlation time) are required, which is often unavailable in practical applications. In contrast, the signal-dependent method has been demonstrated to be more robust to this problem, and thus, has a remarkably better performance. 


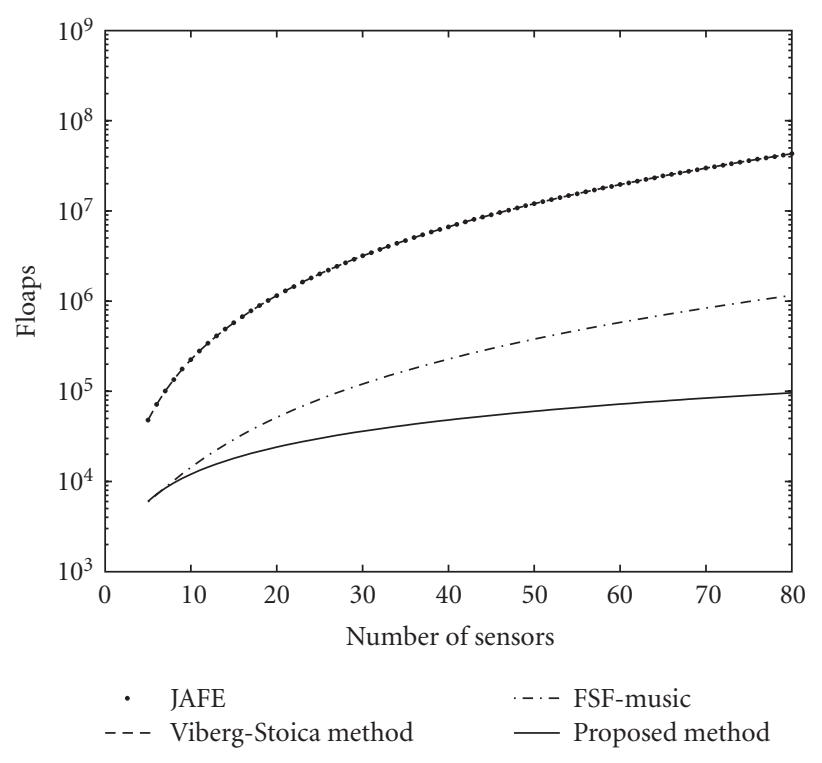

FIGURE 12: Main computational complexity of various algorithms versus the number of sensors with fixed $K=4, N=100$, and $P=3$.

Example 4. In this example, we compare the computational complexities of various algorithms. For easy reference, the comparisons of the computational complexity of these algorithms are summarized in Table 1. More specifically, we consider the case where the source number is $P=3$, the number of snapshots is $N=100$, and temporal smoothing factor is $K=4$. The computational complexity versus the number of sensors is plotted in Figure 12. We observe that the proposed method has the lowest computational complexity among four algorithms.

\subsection{Experimental examples}

We apply the proposed method to the experimental data collected by the real array system. The array system was developed at the research institute of China-Aerospace Science and Industry Corporation (CASIC). The real data was collected in the anechoic chamber on October 20, 2005. The linear frequency modulated (LFM) signals at S-band were used in the experiment. The array system is a horizontal ULA which consists of $M=8$ elements. The spacing between adjacent elements is $4.00 \mathrm{~cm}$. After demodulation to IF, the data was sampled at a rate of $160 \mathrm{MHz}$ with 12-bit precision, and 256 snapshots were collected at each antenna output. There are three uncorrelated sources arriving at the array from $\theta_{1}=3^{\circ}, \theta_{2}=-9^{\circ}$, and $\theta_{3}=8^{\circ}$ with SNRs of $12 \mathrm{~dB}$, $10 \mathrm{~dB}$, and $10 \mathrm{~dB}$, respectively. The transmitted frequencies and the normalized frequencies (after demodulation) of three sources are $(3.090 \mathrm{GHz}, 0.34375),(3.075 \mathrm{GHz}, 0.25)$, and $(3.060 \mathrm{GHz}, 0.15625)$, respectively. The corresponding bandwidths are $6 \mathrm{MHz}, 10 \mathrm{MHz}$, and $7.5 \mathrm{MHz}$, respectively. The source with the direction of $\theta_{1}=3^{\circ}$ is assumed to be the desired signal, and only its transmitted waveform is known a priori.

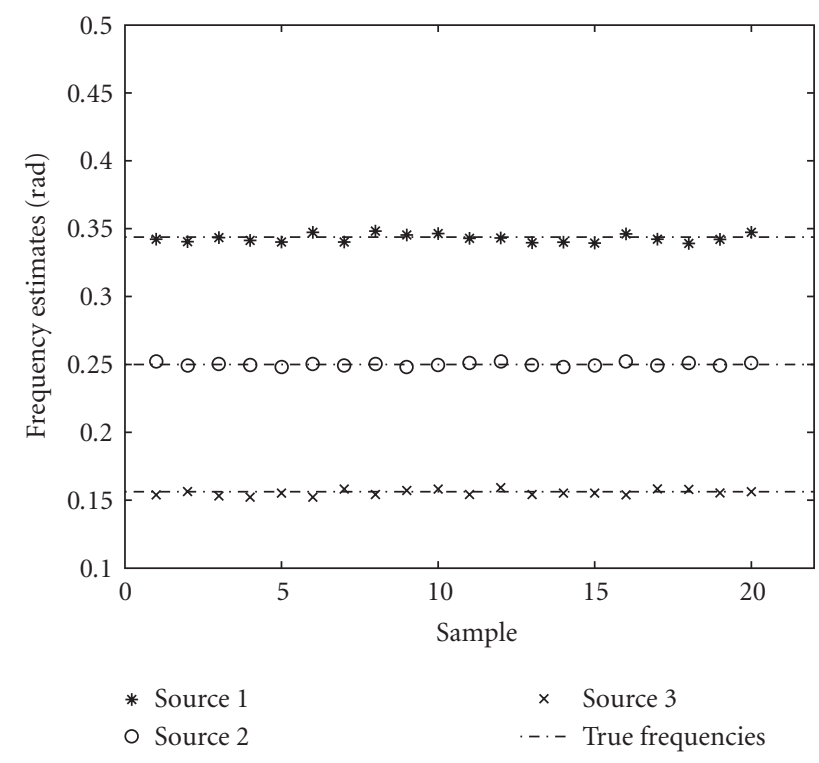

(a)

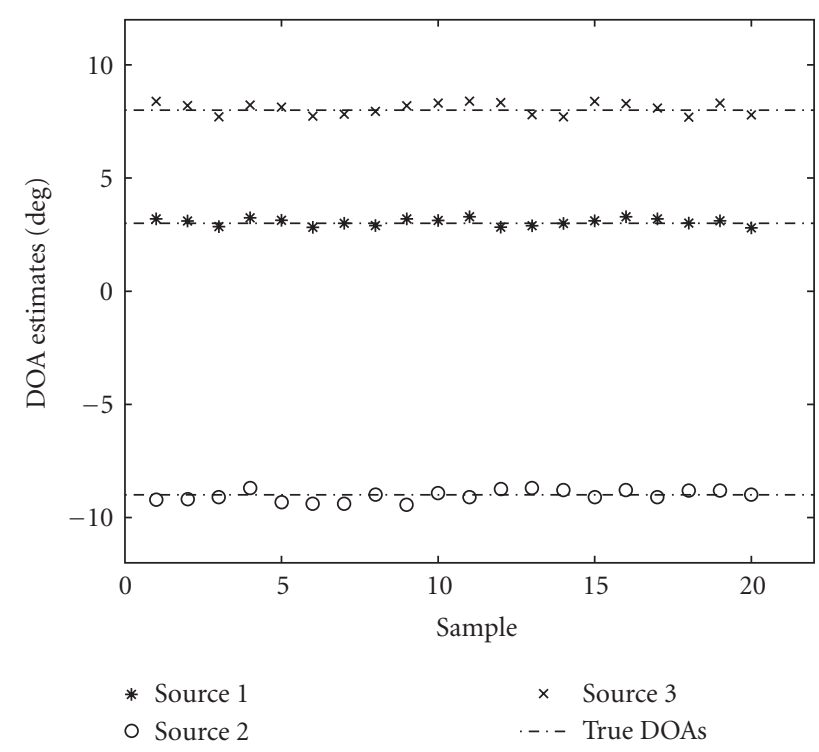

(b)

Figure 13: Frequency and DOA estimates with the proposed methods by using experimental data. $(M=8, K=4, N=256)$. (a) Frequency estimation. (b) DOA estimation.

Figures 13(a) and 13(b) show the experiment results of the proposed method with $K=4$. Each curve contains 20 estimates. From Figure 13, we observe that the estimation performance of the proposed method is reliable in this experiment. Based on the aforementioned 20 estimates, the comparisons of the means and RMSEs with the prewhitened JAFE [5] and the Zoltowski method [7] are made in Table 2. (Due to the difference between two system models, we only test this algorithm under the model of 1-D ULA instead of 2-D L-shaped nonuniform linear array which is used in [7].) Note that for the experimental data analyzed here, the proposed method and the prewhitened JAFE provide similar performances and outperform the Zoltowski method. 


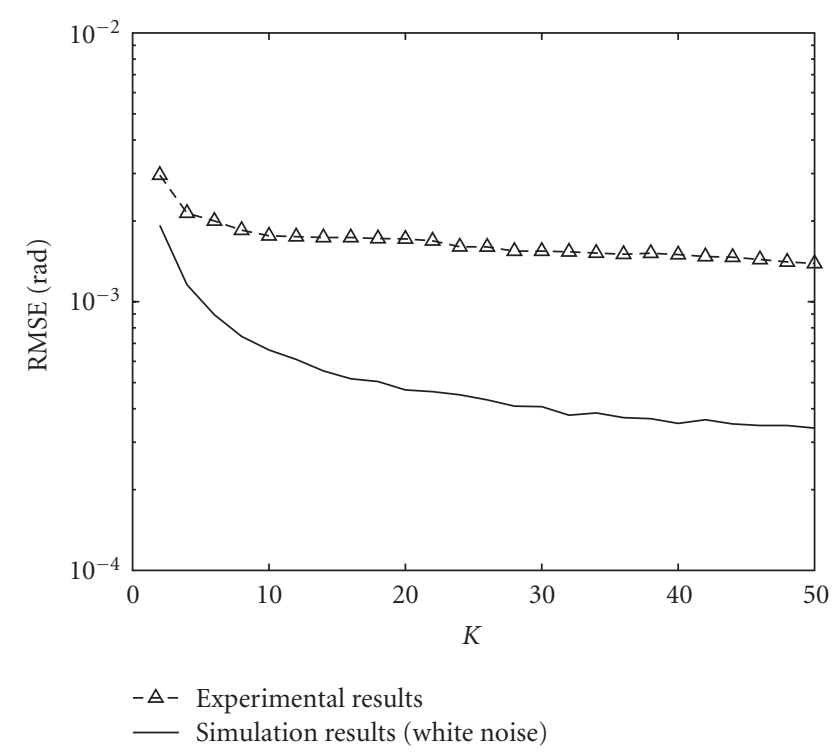

(a)

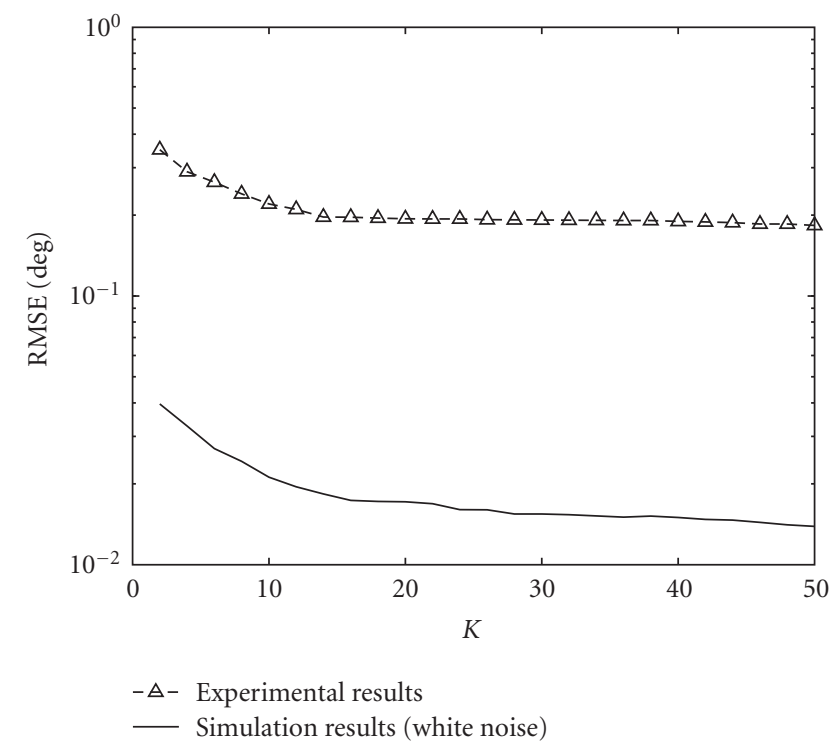

(b)

FIGURE 14: RMSE curves for frequency and DOA estimation of the desired signal as functions of temporal smoothing factor $K .(M=8$, $N=256$ ). (a) Frequency estimation. (b) DOA estimation.

Meanwhile, the effect of temporal smoothing on the RMSE is investigated. In Figures 14(a) and 14(b), we plot the RMSEs of the desired signal as a function of temporal smoothing factor $K$, and show both the experimental results (based on 20 experimental trials) and the simulation results (based on 1000 Monte Carlo trials). As we expect, the RMSEs of frequency and DOA estimation decrease when $K$ changes from 2 to 50 , which is consistent with the discussion in [5]. In [5], the optimum temporal smoothing factors are given, that is, $K=2 N / 3$ for frequency estimation and $K=N / 2$ for DOA estimation, respectively. However, this is the most computationally prohibitive case. From the results in Figures 14(a) and 14(b), we suggest choosing $K$ between 4 and 10 to make a compromise between complexity and accuracy. In addition, from above experimental results, it should be noted that the performance degradation of the experimental results is due to the presence of colored noise and other real-world effects.

\section{CONCLUSION}

To apply the joint DOA and frequency estimation more effectively in practical applications, a robust and computationally efficient method is proposed by using the signaldependent MWF and subspace technique. It is shown that, in contrast to the classical subspace-based methods, the proposed method provides a robust performance in the presence of colored noise. Meanwhile, its computational complexity is much lower than the classical subspace-based methods. It is believed that these advantages can make the proposed method more efficient and feasible in real-world applications.

Finally, it should be noted that this paper does not consider the case of coherent signals. As shown in Figure 7, two sources with the same frequency cannot be resolved by using the proposed method. Following the method of [5], we can resort to the spatial smoothing technique to decorrelate the coherent signals. However, this will result in some disadvantages, such as the increase of the computational complexity and the loss of spatial resolution. We will focus on these problems in our further research.

\section{APPENDICES}

\section{A. PROOF OF PROPERTY 1}

Let $\varphi_{i}=2 \pi d / \lambda_{i} \sin \theta_{i}$, then we have

$$
\begin{aligned}
& \boldsymbol{\Omega}=\left[\begin{array}{c}
\mathbf{A} \\
\mathrm{A} \Phi \\
\vdots \\
\mathrm{A} \Phi^{K-1}
\end{array}\right] \\
& {\left[\begin{array}{cccc}
1 & 1 & \cdots & 1 \\
e^{j \varphi_{1}} & e^{j \varphi_{2}} & & e^{j \varphi_{P}} \\
\vdots & \vdots & & \vdots \\
e^{j(M-1) \varphi_{1}} & e^{j(M-1) \varphi_{2}} & & e^{j(M-1) \varphi_{P}} \\
e^{j \omega_{1}} & e^{j \omega_{2}} & & e^{j \omega_{P}} \\
e^{j \varphi_{1}} e^{j \omega_{1}} & e^{j \varphi_{2}} e^{j \omega_{2}} & & e^{j \varphi_{P}} e^{j \omega_{P}} \\
\vdots & \vdots & & \vdots \\
e^{j(M-1) \varphi_{1}} e^{j \omega_{1}} & e^{j(M-1) \varphi_{2}} e^{j \omega_{2}} & \ddots & e^{j(M-1) \varphi_{P}} e^{j \omega_{P}} \\
\vdots & \vdots & & \vdots \\
e^{j(K-1) \omega_{1}} & e^{j(K-1) \omega_{2}} & & e^{j(K-1) \omega_{P}} \\
e^{j \varphi_{1}} e^{j(K-1) \omega_{1}} & e^{j \varphi_{2}} e^{j(K-1) \omega_{2}} & & e^{j \varphi_{P}} e^{j(K-1) \omega_{P}} \\
\vdots & \vdots & & \vdots \\
e^{j(M-1) \varphi_{1}} & e^{j(M-1) \varphi_{2}} & & e^{j(M-1) \varphi_{P}} \\
\times e^{j(K-1) \omega_{1}} & \times e^{j(K-1) \omega_{2}} & \cdots & \times e^{j(K-1) \omega_{P}}
\end{array}\right]} \\
& =\left[\boldsymbol{\Xi}\left(\theta_{1}, \omega_{1}\right), \boldsymbol{\Xi}\left(\theta_{2}, \omega_{2}\right), \ldots, \boldsymbol{\Xi}\left(\theta_{P}, \omega_{P}\right)\right] \text {, }
\end{aligned}
$$


where

$$
\begin{aligned}
& \boldsymbol{\Xi}\left(\theta_{i}, \omega_{i}\right)=\left[\begin{array}{c}
1 \\
e^{j \varphi_{i}} \\
\vdots \\
e^{j(M-1) \varphi_{i}} \\
e^{j \omega_{i}} \\
e^{j \varphi_{i}} e^{j \omega_{i}} \\
\vdots \\
e^{j(M-1) \varphi_{i}} e^{j \omega_{i}} \\
\vdots \\
e^{j(K-1) \omega_{i}} \\
e^{j \varphi_{i}} e^{j(K-1) \omega_{i}} \\
\vdots \\
e^{j(M-1) \varphi_{i}} e^{j(K-1) \omega_{i}}
\end{array}\right]=\left[\begin{array}{c}
\mathbf{a}\left(\theta_{i}\right) \\
e^{j \omega_{i}} \mathbf{a}\left(\theta_{i}\right) \\
\vdots \\
e^{j(K-1) \omega_{i}} \mathbf{a}\left(\theta_{i}\right)
\end{array}\right] \\
& \\
&=\mathbf{b}\left(\omega_{i}\right) \otimes \mathbf{a}\left(\theta_{i}\right) .
\end{aligned}
$$

This proves Property 1.

\section{B. PROOF OF THEOREM 1}

The log likelihood function of the signal is

$$
\begin{aligned}
\ln L(\boldsymbol{\eta})= & \text { const }-M K N \ln \sigma^{2} \\
& -\frac{1}{\sigma^{2}} \sum_{t=1}^{N}\left[\mathbf{X}_{K}(t)-\mathbf{\Omega} \mathbf{g}(t)\right]^{H}\left[\mathbf{X}_{K}(t)-\mathbf{\Omega} \mathbf{g}(t)\right],
\end{aligned}
$$

where the unknown parameter vector $\boldsymbol{\eta}$ is defined as $\boldsymbol{\eta}=\left[\boldsymbol{\theta}^{T} \boldsymbol{\omega}^{T} \operatorname{Re}\{\mathbf{g}(1)\} \operatorname{Im}\{\mathbf{g}(1)\} \cdots \operatorname{Re}\{\mathbf{g}(N)\} \operatorname{Im}\{\mathbf{g}(N)\} \sigma^{2}\right]$

and $\boldsymbol{\theta}=\left[\begin{array}{lll}\theta_{1} & \cdots & \theta_{P}\end{array}\right]^{T}$ is a $P \times 1$ vector containing the DOAs of the sources.

$\boldsymbol{\omega}=\left[\begin{array}{lll}\omega_{1} & \cdots & \omega_{P}\end{array}\right]^{T}$ is a $P \times 1$ vector containing the frequencies of the sources.

The Fisher information matrix (FIX) is given by [32, Appendix B]

$$
\begin{aligned}
\boldsymbol{F I M}(\boldsymbol{\eta})= & E\left\{\left[\frac{\partial \ln L(\boldsymbol{\eta})}{\partial \boldsymbol{\eta}}\right]\left[\frac{\partial \ln L(\boldsymbol{\eta})}{\partial \boldsymbol{\eta}}\right]^{T}\right\} \\
= & {\left[\begin{array}{cccccccc}
\Upsilon_{\boldsymbol{\theta} \boldsymbol{\theta}} & \Upsilon_{\boldsymbol{\theta} \boldsymbol{\omega}} & \boldsymbol{\mu}^{T}(1) & \boldsymbol{\mu}^{T}(2) & \cdots & \boldsymbol{\mu}^{T}(N) & 0 \\
\Upsilon_{\boldsymbol{\omega} \boldsymbol{\theta}} & \Upsilon_{\boldsymbol{\omega} \boldsymbol{\omega}} & \boldsymbol{\nu}^{T}(1) & \boldsymbol{\nu}^{T}(2) & \cdots & \boldsymbol{\nu}^{T}(N) & 0 \\
\boldsymbol{\mu}(1) & \boldsymbol{\nu}(1) & \boldsymbol{\Lambda} & 0 & \cdots & 0 & 0 \\
\boldsymbol{\mu}(2) & \boldsymbol{v}(2) & 0 & \boldsymbol{\Lambda} & \cdots & 0 & 0 \\
\vdots & \vdots & \vdots & \vdots & \ddots & \vdots & 0 \\
\boldsymbol{\mu}(N) & \boldsymbol{v}(N) & 0 & 0 & \cdots & \boldsymbol{\Lambda} & 0 \\
0 & 0 & 0 & 0 & 0 & 0 & \mathrm{CRB}_{\sigma^{2}}^{-1}
\end{array}\right] }
\end{aligned}
$$

where

$$
\begin{gathered}
\Upsilon_{\boldsymbol{\theta} \boldsymbol{\theta}}=\frac{2}{\sigma^{2}} \sum_{t=1}^{N} \operatorname{Re}\left\{\mathbf{G}^{H}(t) \mathbf{D}_{\boldsymbol{\theta}}^{H} \mathbf{D}_{\boldsymbol{\theta}} \mathbf{G}(t)\right\}, \\
\Upsilon_{\boldsymbol{\theta} \boldsymbol{\omega}}=\frac{2}{\sigma^{2}} \sum_{t=1}^{N} \operatorname{Re}\left\{\mathbf{G}^{H}(t) \mathbf{D}_{\boldsymbol{\theta}}^{H} \mathbf{D}_{\boldsymbol{\omega}} \mathbf{G}(t)\right\}, \\
\Upsilon_{\boldsymbol{\omega} \boldsymbol{\theta}}=\frac{2}{\sigma^{2}} \sum_{t=1}^{N} \operatorname{Re}\left\{\mathbf{G}^{H}(t) \mathbf{D}_{\boldsymbol{\omega}}^{H} \mathbf{D}_{\boldsymbol{\theta}} \mathbf{G}(t)\right\}, \\
\Upsilon_{\boldsymbol{\omega} \boldsymbol{\omega}}=\frac{2}{\sigma^{2}} \sum_{t=1}^{N} \operatorname{Re}\left\{\mathbf{G}^{H}(t) \mathbf{D}_{\boldsymbol{\omega}}^{H} \mathbf{D}_{\boldsymbol{\omega}} \mathbf{G}(t)\right\}, \\
\boldsymbol{\Lambda}=\frac{2}{\sigma^{2}}\left[\begin{array}{ll}
\operatorname{Re}\left\{\boldsymbol{\Omega}^{H} \boldsymbol{\Omega}\right\} & -\operatorname{Im}\left\{\boldsymbol{\Omega}^{H} \boldsymbol{\Omega}\right\} \\
\operatorname{Im}\left\{\boldsymbol{\Omega}^{H} \boldsymbol{\Omega}\right\} & \operatorname{Re}\left\{\boldsymbol{\Omega}^{H} \boldsymbol{\Omega}\right\}
\end{array}\right], \\
\boldsymbol{\mu}(t)=\frac{2}{\sigma^{2}}\left[\begin{array}{l}
\operatorname{Re}\left\{\boldsymbol{\Omega}^{H} \mathbf{D}_{\boldsymbol{\theta}} \mathbf{G}(t)\right\} \\
\operatorname{Im}\left\{\boldsymbol{\Omega}^{H} \mathbf{D}_{\boldsymbol{\theta}} \mathbf{G}(t)\right\}
\end{array}\right],
\end{gathered}
$$

$$
\begin{gathered}
\boldsymbol{v}(t)=\frac{2}{\sigma^{2}}\left[\begin{array}{c}
\operatorname{Re}\left\{\boldsymbol{\Omega}^{H} \mathbf{D}_{\boldsymbol{\omega}} \mathbf{G}(t)\right\} \\
\operatorname{Im}\left\{\boldsymbol{\Omega}^{H} \mathbf{D}_{\boldsymbol{\omega}} \mathbf{G}(t)\right\}
\end{array}\right], \\
\mathrm{CRB}_{\sigma^{2}}=\frac{\sigma^{4}}{M K N} .
\end{gathered}
$$

Then, the inverse CRB matrix for $\boldsymbol{\theta}$ and $\boldsymbol{\omega}$ is obtained by the following:

$$
\begin{aligned}
& \mathrm{CRB}^{-1}(\boldsymbol{\theta}, \boldsymbol{\omega}) \\
& =\left[\begin{array}{ll}
\Upsilon_{\boldsymbol{\theta} \theta} & \Upsilon_{\boldsymbol{\theta \omega}} \\
\Upsilon_{\boldsymbol{\omega} \boldsymbol{\theta}} & \Upsilon_{\boldsymbol{\omega} \boldsymbol{\omega}}
\end{array}\right]-\left[\begin{array}{llll}
\boldsymbol{\mu}^{T}(1) & \boldsymbol{\mu}^{T}(2) & \cdots & \boldsymbol{\mu}^{T}(N) \\
\boldsymbol{\nu}^{T}(1) & \boldsymbol{\nu}^{T}(2) & \cdots & \boldsymbol{\nu}^{T}(N)
\end{array}\right] \\
& {\left[\begin{array}{cccc}
\Lambda & 0 & \cdots & 0 \\
0 & \Lambda & \cdots & 0 \\
\vdots & \vdots & \Lambda & \vdots \\
0 & 0 & \cdots & \boldsymbol{\Lambda}
\end{array}\right]^{-1}\left[\begin{array}{cc}
\boldsymbol{\mu}(1) & \boldsymbol{\nu}(1) \\
\boldsymbol{\mu}(2) & \boldsymbol{\nu}(2) \\
\vdots & \vdots \\
\boldsymbol{\mu}(N) & \boldsymbol{\nu}(N)
\end{array}\right]} \\
& =\left[\begin{array}{ll}
\Upsilon_{\boldsymbol{\theta}} & \Upsilon_{\boldsymbol{\theta} \boldsymbol{\omega}} \\
\Upsilon_{\boldsymbol{\omega} \boldsymbol{\theta}} & \Upsilon_{\boldsymbol{\omega} \boldsymbol{\omega}}
\end{array}\right]-\sum_{t=1}^{N}\left[\begin{array}{c}
\boldsymbol{\mu}^{T}(t) \\
\boldsymbol{\nu}^{T}(t)
\end{array}\right] \boldsymbol{\Lambda}^{-1}\left[\begin{array}{ll}
\boldsymbol{\mu}(t) & \boldsymbol{\nu}(t)
\end{array}\right] \\
& =\frac{2}{\sigma^{2}} \sum_{t=1}^{N} \operatorname{Re}\left\{\left[\begin{array}{cc}
\mathbf{G}^{H}(t) & 0 \\
0 & \mathbf{G}^{H}(t)
\end{array}\right]\left[\begin{array}{l}
\mathbf{D}_{\boldsymbol{\theta}}^{H} \\
\mathbf{D}_{\boldsymbol{\omega}}^{H}
\end{array}\right]\right. \\
& \left.\cdot\left[\mathbf{I}-\boldsymbol{\Omega}\left(\boldsymbol{\Omega}^{H} \boldsymbol{\Omega}\right)^{-1} \boldsymbol{\Omega}^{H}\right]\left[\begin{array}{ll}
\mathbf{D}_{\boldsymbol{\theta}} & \mathbf{D}_{\boldsymbol{\omega}}
\end{array}\right]\left[\begin{array}{cc}
\mathbf{B}(t) & 0 \\
0 & \mathbf{B}(t)
\end{array}\right]\right\} \\
& =\frac{2}{\sigma^{2}} \sum_{t=1}^{N} \operatorname{Re}\left\{\mathbf{Z}^{H}(t) \mathbf{D}^{H} \mathbf{P}_{\mathbf{\Omega}}^{\perp} \mathbf{D Z}(t)\right\} .
\end{aligned}
$$

This concludes the proof. 


\section{PROOF OF THEOREM 2}

Let $\boldsymbol{\Gamma}=\mathbf{D}^{H} \mathbf{P}_{\Omega}^{\perp} \mathbf{D}$, we have

$$
\lim _{N \rightarrow \infty} \sum_{t=1}^{N} \operatorname{Re}\left\{\mathbf{Z}^{H}(t) \mathbf{\Gamma} \mathbf{Z}(t)\right\}=\lim _{N \rightarrow \infty} \operatorname{Re}\left\{\left[\sum_{t=1}^{N} \mathbf{z}^{H}(t) \mathbf{z}(t)\right] \odot \mathbf{\Gamma}\right\},
$$

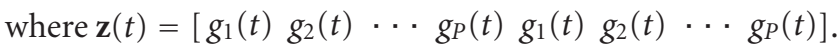
For large $N$,

$$
\lim _{N \rightarrow \infty} \sum_{t=1}^{N} \mathbf{z}^{H}(t) \mathbf{z}(t) \approx N \cdot\left[\begin{array}{ll}
\mathbf{R}_{\mathbf{g}}^{T} & \mathbf{R}_{\mathbf{g}}^{T} \\
\mathbf{R}_{\mathbf{g}}^{T} & \mathbf{R}_{\mathbf{g}}^{T}
\end{array}\right]=N \cdot \mathfrak{R}^{T},
$$

where $\mathbf{R}_{\mathbf{g}}=\lim _{N \rightarrow \infty}(1 / N) \sum_{t=1}^{N} \mathbf{g}(t) \mathbf{g}^{H}(t)$. Substituting (C.1) and (C.2) into (20), we obtain (22). This concludes the proof.

\section{ACKNOWLEDGMENTS}

The authors are grateful to the Department of Electronic Engineering, Nanjing University of Science and Technology, for permission to use the experimental data. The authors would also like to thank the anonymous reviewers for their useful comments and insightful suggestions on a former version of this paper.

\section{REFERENCES}

[1] M. P. Clark and L. L. Scharf, "A maximum likelihood estimation technique for spatial-temporal modal analysis," in Proceedings of the 25th Asilomar Conference on Signals, Systems and Computers, vol. 1, pp. 257-261, Pacific Grove, Calif, USA, November 1991.

[2] M. P. Clark and L. L. Scharf, "Two-dimensional modal analysis based on maximum likelihood," IEEE Transactions on Signal Processing, vol. 42, no. 6, pp. 1443-1452, 1994.

[3] M. Wax, T.-J. Shan, and T. Kailath, "Spatio-temporal spectral analysis by eigenstructure methods," IEEE Transactions on Acoustics, Speech, and Signal Processing, vol. 32, no. 4, pp. 817827, 1984.

[4] A. N. Lemma, A.-J. van der Veen, and E. F. Deprettere, "Joint angle-frequency estimation using multi-resolution ESPRIT," in Proceedings of IEEE International Conference on Acoustics, Speech and Signal Processing (ICASSP '98), vol. 4, pp. 19571960, Seattler, Wash, USA, May 1998.

[5] A. N. Lemma, A.-J. van der Veen, and E. F. Deprettere, "Analysis of joint angle-frequency estimation using ESPRIT," IEEE Transactions on Signal Processing, vol. 51, no. 5, pp. 12641283, 2003.

[6] M. Viberg and P. Stoica, "A computationally efficient method for joint direction finding and frequency estimation in colored noise," in Proceedings of the 32nd Asilomar Conference on Signals, Systems and Computers, vol. 2, pp. 1547-1551, Pacific Grove, Calif, USA, November 1998.

[7] M. D. Zoltowski and C. P. Mathews, "Real-time frequency and 2-D angle estimation with sub-Nyquist spatio-temporal sampling," IEEE Transactions on Signal Processing, vol. 42, no. 10, pp. 2781-2794, 1994.

[8] M. Haardt and J. A. Nossek, "3-D unitary ESPRIT for joint 2$\mathrm{D}$ angle and carrier estimation," in Proceedings of IEEE International Conference on Acoustics, Speech, and Signal Processing
(ICASSP '97), vol. 1, pp. 255-258, Munich, Germany, April 1997.

[9] J.-D. Lin, W.-H. Fang, Y.-Y. Wang, and J.-T. Chen, "FSF MUSIC for joint DOA and frequency estimation and its performance analysis," IEEE Transactions on Signal Processing, vol. 54, no. 12, pp. 4529-4542, 2006.

[10] H. Belkacemi and S. Marcos, "Robust subspace-based algorithms for joint angle/Doppler estimation in non-Gaussian clutter," Signal Processing, vol. 87, no. 7, pp. 1547-1558, 2007.

[11] R. Schmidt, A signal subspace approach to multiple emitter location and spectral estimation, Ph.D. thesis, Stanford University, Stanford, Calif, USA, November 1981.

[12] R. Roy, ESPRIT-estimation of signal parameters via rotational invariance techniques, Ph.D. thesis, Stanford University, Stanford, Calif, USA, August 1987.

[13] J. Li and R. T. Compton Jr., "Maximum likelihood angle estimation for signals with known waveforms," IEEE Transactions on Signal Processing, vol. 41, no. 9, pp. 2850-2862, 1993.

[14] J. Li, B. Halder, P. Stoica, and M. Viberg, "Computationally efficient angle estimation for signals with known waveforms," IEEE Transactions on Signal Processing, vol. 43, no. 9, pp. 21542163, 1995.

[15] M. Wax and A. Leshem, "Joint estimation of time delays and directions of arrival of multiple reflections of a known signal," IEEE Transactions on Signal Processing, vol. 45, no. 10, pp. 2477-2484, 1997.

[16] A. L. Swindlehurst, "Time delay and spatial signature estimation using known asynchronous signals," IEEE Transactions on Signal Processing, vol. 46, no. 2, pp. 449-462, 1998.

[17] A. Jakobsson, A. L. Swindlehurst, and P. Stoica, "Subspacebased estimation of time delays and doppler shifts," IEEE Transactions on Signal Processing, vol. 46, no. 9, pp. 2472-2483, 1998.

[18] F. Gini, M. Greco, and A. Farina, "Multiple radar targets estimation by exploiting induced amplitude modulation," IEEE Transactions on Aerospace and Electronic Systems, vol. 39, no. 4, pp. 1316-1332, 2003.

[19] J. Ward and R. T. Compton Jr., "Improving the performance of a slotted ALOHA packet radio network with an adaptive array," IEEE Transactions on Communications, vol. 40, no. 2, pp. 292-300, 1992.

[20] Y. Jiang, P. Stoica, and J. Li, "Array signal processing in the known waveform and steering vector case," IEEE Transactions on Signal Processing, vol. 52, no. 1, pp. 23-35, 2004.

[21] J. S. Goldstein, I. S. Reed, and L. L. Scharf, "A multistage representation of the Wiener filter based on orthogonal projections," IEEE Transactions on Information Theory, vol. 44, no. 7, pp. 2943-2959, 1998.

[22] J. R. Guerci, J. S. Goldstein, P. A. Zulch, and I. S. Reed, "Optimal reduced-rank 3D STAP for joint hot and cold clutter mitigation," in Proceedings of the 11th IEEE Radar Conference, pp. 119-124, Waltham, Mass, USA, April 1999.

[23] M. L. Honig and W. Xiao, "Performance of reduced-rank linear interference suppression," IEEE Transactions on Information Theory, vol. 47, no. 5, pp. 1928-1946, 2001.

[24] J. R. Guerci and J. S. Bergin, "Principal components, covariance matrix tapers, and the subspace leakage problem," IEEE Transactions on Aerospace and Electronic Systems, vol. 38, no. 1, pp. 152-162, 2002.

[25] M. Wax and T. Kailath, "Detection of signals by information theoretic criteria," IEEE Transactions on Acoustics, Speech, and Signal Processing, vol. 33, no. 2, pp. 387-392, 1985. 
[26] J. Ward, "Space-time adaptive processing for airborne radar," Tech. Rep. 1015, Lincoln Laboratory, Lexington, Mass, USA, December 1994.

[27] J. Roe, S. Cussons, and A. Feltham, "Knowledge-based signal processing for radar ESM systems," IEE proceedings F, vol. 137, no. 5, pp. 293-301, 1990.

[28] J. B. Tsui, Microwave Receivers with Electronic Warfare Applications, John Wiley \& Sons, New York, NY, USA, 1986.

[29] D. C. Ricks and J. S. Goldstein, "Efficient architectures for implementing adaptive algorithms," in Proceedings of the Antenna Applications Symposium, pp. 29-41, Monticello, Ill, USA, September 2000.

[30] P. Stoica and A. Nehorai, "MUSIC, maximum likelihood, and Cramér-Rao bound," IEEE Transactions on Acoustics, Speech, and Signal Processing, vol. 37, no. 5, pp. 720-741, 1989.

[31] P. Stoica and A. Nehorai, "Performance study of conditional and unconditional direction-of-arrival estimation," IEEE Transactions on Acoustics, Speech, and Signal Processing, vol. 38, no. 10, pp. 1783-1795, 1990.

[32] P. Stoica and R. L. Moses, Introduction to Spectral Analysis, Prentice-Hall, Englewood Cliffs, NJ, USA, 1997.

[33] H. L. Van Trees, Optimun Array Processing, Wiley-Interscience, New York, NY, USA, 2002.

[34] H. Ye and R. D. Degroat, "Maximum likelihood DOA estimation and asymptotic Cramér-Rao bounds for additive unknown colored noise," IEEE Transactions on Signal Processing, vol. 43, no. 4, pp. 938-949, 1995.

[35] J. H. Michels, P. K. Varshney, and D. D. Weiner, "Synthesis of correlated multichannel random processes," IEEE Transactions on Signal Processing, vol. 42, no. 2, pp. 367-375, 1994.

[36] R. A. Horn and C. R. Johnson, Matrix Analysis, Cambridge University Press, Cambridge, UK, 1985. 